\title{
Broadband dielectric spectroscopy of glucose aqueous solution: Analysis of the hydration state and the hydrogen bond network.
}

\section{$\operatorname{AUTHOR}(S)$ :}

Shiraga, Keiichiro; Suzuki, Tetsuhito; Kondo, Naoshi; Tajima, Takuro; Nakamura, Masahito; Togo, Hiroyoshi; Hirata, Akihiko; Ajito, Katsuhiro; Ogawa, Yuichi

\section{CITATION:}

Shiraga, Keiichiro ...[et al]. Broadband dielectric spectroscopy of glucose aqueous solution: Analysis of the hydration state and the hydrogen bond network.. The Journal of chemical physics 2015, 142(23): 234504.

\section{ISSUE DATE:}

2015-06-19

\section{URL:}

http://hdl.handle.net/2433/201613

\section{RIGHT:}

(C) 2015 American Institute of Physics. This article may be downloaded for personal use only. Any other use requires prior permission of the author and the American Institute of Physics. 


\section{AIP | The Journal of}

\section{Broadband dielectric spectroscopy of glucose aqueous solution: Analysis of the hydration state and the hydrogen bond network}

Keiichiro Shiraga, Tetsuhito Suzuki, Naoshi Kondo, Takuro Tajima, Masahito Nakamura, Hiroyoshi Togo,

Akihiko Hirata, Katsuhiro Ajito, and Yuichi Ogawa

Citation: The Journal of Chemical Physics 142, 234504 (2015); doi: 10.1063/1.4922482

View online: http://dx.doi.org/10.1063/1.4922482

View Table of Contents: http://scitation.aip.org/content/aip/journal/jcp/142/23?ver=pdfcov

Published by the AIP Publishing

\section{Articles you may be interested in}

Hydrogen bond network in the hydration layer of the water confined in nanotubes increasing the dielectric constant parallel along the nanotube axis

J. Chem. Phys. 143, 114708 (2015); 10.1063/1.4931414

Hydration and rotational diffusion of levoglucosan in aqueous solutions

J. Chem. Phys. 140, 184505 (2014); 10.1063/1.4873575

Terahertz absorption of dilute aqueous solutions

J. Chem. Phys. 137, 235103 (2012); 10.1063/1.4772000

Structural properties of glucose-dimethylsulfoxide solutions probed by Raman spectroscopy

J. Chem. Phys. 130, 164501 (2009); 10.1063/1.3116250

Hydrogen bond dynamics and water structure in glucose-water solutions by depolarized Rayleigh scattering and low-frequency Raman spectroscopy

J. Chem. Phys. 127, 024504 (2007); 10.1063/1.2748405

\section{AlP $\mid$ APL Photonics}

APL Photonics is pleased to announce Benjamin Eggleton as its Editor-in-Chief

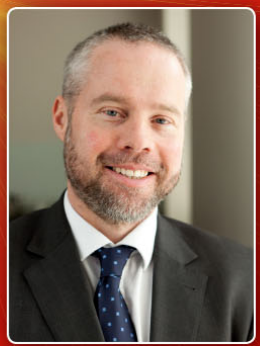




\title{
Broadband dielectric spectroscopy of glucose aqueous solution: Analysis of the hydration state and the hydrogen bond network
}

\author{
Keiichiro Shiraga, ${ }^{1}$ Tetsuhito Suzuki, ${ }^{1}$ Naoshi Kondo, ${ }^{1}$ Takuro Tajima, ${ }^{2}$ \\ Masahito Nakamura, ${ }^{2}$ Hiroyoshi Togo, ${ }^{3}$ Akihiko Hirata, ${ }^{2}$ Katsuhiro Ajito, ${ }^{2}$ \\ and Yuichi Ogawa ${ }^{1, a)}$ \\ ${ }^{1}$ Graduate School of Agriculture, Kyoto University, Kitashirakawa-oiwakecho, Sakyo-ku, \\ Kyoto 606-8502, Japan \\ ${ }^{2}$ NTT Device Technology Laboratories, NTT Corporation, Morinosato Wakamiya, Atsugi-shi, \\ Kanagawa 243-0198, Japan \\ ${ }^{3}$ Science and Core Technology Laboratories, NTT Corporation, Morinosato Wakamiya, Atsugi-shi, \\ Kanagawa 243-0198, Japan
}

(Received 8 March 2015; accepted 1 June 2015; published online 19 June 2015)

\begin{abstract}
Recent studies of saccharides' peculiar anti-freezing and anti-dehydration properties point to a close association with their strong hydration capability and destructuring effect on the hydrogen bond (HB) network of bulk water. The underlying mechanisms are, however, not well understood. In this respect, examination of the complex dielectric constants of saccharide aqueous solutions, especially over a broadband frequency region, should provide interesting insights into these properties, since the dielectric responses reflect corresponding dynamics over the time scales measured. In order to do this, the complex dielectric constants of glucose solutions between $0.5 \mathrm{GHz}$ and $12 \mathrm{THz}$ (from the microwave to the far-infrared region) were measured. We then performed analysis procedures on this broadband spectrum by decomposing it into four Debye and two Lorentz functions, with particular attention being paid to the $\beta$ relaxation (glucose tumbling), $\delta$ relaxation (rotational polarization of the hydrated water), slow relaxation (reorientation of the HB network water), fast relaxation (rotation of the non-HB water), and intermolecular stretching vibration (hindered translation of water). On the basis of this analysis, we revealed that the hydrated water surrounding the glucose molecules exhibits a mono-modal relaxational dispersion with 2-3 times slower relaxation times than unperturbed bulk water and with a hydration number of around 20. Furthermore, other species of water with distorted tetrahedral HB water structures, as well as increases in the relative proportion of non-HB water molecules which have a faster relaxation time and are not a part of the surrounding bulk water HB network, was found in the vicinity of the glucose molecules. These clearly point to the HB destructuring effect of saccharide solutes in aqueous solution. The results, as a whole, provide a detailed picture of glucose-water and water-water interactions in the vicinity of the glucose molecules at various time scales from sub-picosecond to hundreds of picoseconds. (C) 2015 AIP Publishing LLC. [http://dx.doi.org/10.1063/1.4922482]
\end{abstract}

\section{INTRODUCTION}

Saccharides, in conjunction with water, play an important role in various biological processes, including structural support $^{1}$ and molecular recognition. ${ }^{2}$ Moreover, saccharides' role as a bioprotectant agent in promoting anti-freezing and dehydration properties has attracted wide attention. ${ }^{3}$ Recent studies suggest, both computationally ${ }^{4-7}$ and experimentally, ${ }^{8-10}$ that these peculiar properties of the saccharides arise from changes in the structural and dynamical properties of the water surrounding the saccharide molecules when in an aqueous phase. The abundant polar hydroxyl groups, with their strong dipole interaction capability, in the saccharides are thought to alter the local tetrahedral coordination of the hydrogen bond (HB) network of water in the vicinity of the saccharide

\footnotetext{
a) Author to whom correspondence should be addressed. Electronic mail: ogawayu@kais.kyoto-u.ac.jp. Fax: +81757536171.
}

molecules. In particular, a recent hypothesis links the bioprotective properties of saccharides to their strong modification of the tetrahedral structure of water. ${ }^{6,8,9}$ Therefore, detailed experimental observations of the HB network of water in the vicinity of saccharide solutions are essential for understanding the underlying mechanisms for some of the saccharides' chemical and physical properties.

The HBs of the water surrounding polar groups are believed to have a distorted three-dimensional coordination, as well as having their reorientation dynamically retarded. ${ }^{11}$ The importance of such water molecules perturbed by polar groups (hydrated water), whose structural and dynamical characteristics differ from that of bulk water, has frequently been emphasized in biological processes. ${ }^{12,13}$ Although numerous experimental approaches have been used to quantitatively estimate the hydration state of saccharides, the underlying mechanisms are still debated due to the ambiguous, piecemeal, and model-dependent definition of hydrated water. 
Additionally, recent molecular dynamics (MD) simulations ${ }^{6}$ and Raman spectroscopy ${ }^{8,10}$ point to the disruption of the HB network structure of water, distorting the tetrahedral coordination of water to their nearest HB partners. This phenomenon is referred to as the "destructuring effect" on the proximal water HB network and has been put forward as a partial explanation for the peculiar bioprotective action of saccharides. Therefore, despite the traditionally accepted "structuremaking" aspect of saccharides, mainly due to the larger number of hydrated water molecules, saccharides also have a weak and local "structure-breaking" aspect. However, without further experimental evidence regarding this destructuring effect, it is difficult to clarify the exact relationship to the bioprotective characteristics of saccharides.

Although the results from a number of experimental methods, including the Raman spectroscopy, ${ }^{8-10}$ depolarized light scattering, ${ }^{14-18}$ nuclear magnetic resonance (NMR), ${ }^{19}$ neutron diffraction, ${ }^{20}$ infrared (IR) spectroscopy, ${ }^{9}$ polarizationresolved femtosecond spectroscopy, ${ }^{21}$ and dielectric spectroscopy ${ }^{22-24}$ have been compared to those from MD simulations to characterize the hydration state and the destructuring effect of saccharides in aqueous solution, each has its limitations. While neutron diffraction provides structural information, such as distance and distribution, through a radial distribution function (RDF), it conveys few dynamical properties of the water around solutes. In NMR measurements, one of the most commonly used methods to investigate the hydration state (i.e., hydration number and degree of retardation) around solutes, ${ }^{2} \mathrm{H}$ or ${ }^{17} \mathrm{O}$ substitution is required, in order to distinguish small solute-induced changes and avoid the intermolecular nuclei Overhauser effects between water and solutes. ${ }^{19,25}$ However, isotopic substitution can lead to a slight modulation of the HB network structure, ${ }^{26,27}$ risking the misinterpretation of native hydration phenomenon in saccharides. Above all, the long correlation time of NMR (not shorter than $1 \mathrm{~ns}$ ) obscures the detailed HB dynamics, which typically occur on a picosecond time scale. ${ }^{28}$ On the other hand, Raman and IR spectroscopies have focused on the $\mathrm{O}-\mathrm{H}$ stretching vibration mode around $100 \mathrm{THz}$. This anharmonic $\mathrm{O}-\mathrm{H}$ stretching mode of water redshifts in the presence of intermolecular HBs, acting on the intramolecular degrees of freedom, and thus, its spectrum shape is related to the distribution of HBs, such as the mean HB number and distance. ${ }^{8,29}$ In order to distinguish the $\mathrm{O}-\mathrm{H}$ vibration of water molecules and of the hydroxyl group inside the saccharide solutes in these Raman and IR measurements, it is necessary to make unsubstantiated assumptions, thus making it difficult to quantitatively discuss the hydration and destructuring effect. Consequently, while these different techniques provide different pieces of the puzzle, they do not provide a coherent overall picture, fueling continued controversy about proximal water structure and dynamics around saccharide solutes.

On the other hand, terahertz (THz) spectroscopy is a reliable technique to directly probe the intermolecular dynamics of water and has the potential to provide experimental evidence and achieve consensus on the increasingly controversial issue of water structure. Furthermore, in recent studies, dielectric spectroscopy in the $\mathrm{THz}$ region has attracted attention as a means to elucidate solute-induced water interactions. ${ }^{30,31}$
Moreover, it has been found that THz spectroscopy can be used to evaluate the global hydration state of saccharides extending up to $5 \AA$ from the solute surface (beyond the first hydration shell). ${ }^{32,33}$ It also has the potential to analyze the population of non-HB water transiently freed from the bulk water HB network and the disrupted tetrahedral water coordination, ${ }^{34,35}$ which may provide direct evidence of the destructuring effect on the water HB network. Hence, the dielectric responses in the $\mathrm{THz}$ frequency range are a prospective tool to investigate the hydration state and destructuring effect of saccharides on the proximal HB water network. This will though require a broadband measurement over the microwave- $\mathrm{THz}-$ farIR region, as the $\mathrm{THz}$ spectrum of aqueous solution consists of the superposition of several broad components, ${ }^{34,35}$ unlike IR absorption bands where each band is separated from one another.

In this study, we measure the complex dielectric constant, $\tilde{\varepsilon}(\omega)=\operatorname{Re}[\varepsilon(\omega)]-i \operatorname{Im}[\varepsilon(\omega)]$, of water and aqueous saccharide solution from $0.5 \mathrm{GHz}$ to $12 \mathrm{THz}$. This allows us to accurately decompose the complex dielectric constant in the $\mathrm{THz}$ region into its constituents. Armed with these results, the hydration state and destructuring effect of saccharides are discussed in detail. In terms of hydration state, we evaluate both the hydration number and the orientational direction retardation of the hydrated water compared to that of bulk water. Results from the population of the non-HB water and the distortion of the tetrahedral $\mathrm{HB}$ coordination will be used to investigate the destructuring effect on the water HB network. D-glucose, a canonical saccharide prototype, was chosen as the model saccharide sample to measure, due to its simple chemical structure and the abundant reported values in the literature to compare with our results.

\section{EXPERIMENTAL SECTION}

\section{A. Sample preparation}

$\mathrm{D}(+)$-glucose powder ( $\geq 98.0 \%$ purity, Wako Pure Chemical Industry, Ltd.) was dissolved into pure water, demineralized by a Millipore Elix3 UV (Millipore, Ltd.), at $0.146 \mathrm{M}-1.462 \mathrm{M}$ (2.59-24.05 in wt. \%). All solutions were stored at $277 \mathrm{~K}$ for at least $24 \mathrm{~h}$ prior to the measurement, in order to permit the establishment of an anomeric equilibrium.

\section{B. Determination of the complex dielectric constants}

The complex dielectric constant of distilled water and the glucose aqueous solutions was determined from $0.5 \mathrm{GHz}$ to $12 \mathrm{THz}$ (with a gap between $40 \mathrm{GHz}$ and $0.25 \mathrm{THz}$ ), by combining a vector network analyzer (VNA), a THz timedomain attenuated total reflection (THz TD-ATR) spectroscopy, and a far-IR Fourier transform attenuated total reflection (FIR FT-ATR) spectroscopy.

The system used to determine the complex dielectric constant from 0.5 to $40 \mathrm{GHz}$ is an Agilent 85070E Openended Coaxial Probe (Agilent, Ltd.) connected to an Agilent E8362B Vector Network Analyzer (Agilent, Ltd.). The VNA software calculated the real and imaginary parts of the complex 
dielectric constant from the $\mathrm{S}$ parameters by immersing the probe into the sample solution fixed at $300 \pm 0.8 \mathrm{~K}$, after calibrating with three different loads: air, short-circuit, and ultrapure water (18 M $\Omega$ ). In order to understand how well our commercial network analyzer along with commercial VNA software was able to reproduce well-known spectra of a reference liquid sample, the measured complex dielectric constant of water was compared with that reported in a previous study. ${ }^{36}$ The resulting deviation of the real and imaginary parts was less than 0.5 and 0.4 , respectively, ensuring a reliability of our VNA measurement system.

For $0.25-3.0 \mathrm{THz}$ measurements, a commercial THz TDATR spectrometer, TAS7500SP (Advantest, Ltd.), consisting of an ATR prism made of silicon was used. The temperature controller was attached to the ATR prism to keep the sample temperature at $300 \pm 0.1 \mathrm{~K}$. In this system, optical excitations were applied by two mode-locked Ti:sapphire lasers, emitting at $800 \mathrm{~nm}$ wavelength with a 40 fs pulse width. Two fs lasers were linked at a slightly different repetition frequency and served as a pump and a probe light, respectively; this scheme, a so-called asynchronous optical sampling strategy, ${ }^{37,38}$ actualizes rapid scanning unlimited by the mechanical delay stage. The pump pulse was focused on the biased InGaAs photoconductive antenna to emit a time-domain $\mathrm{THz}$ pulse. The $\mathrm{THz}$ pulse was collimated by an off-axis parabolic mirror and was separated into two lines by a beam splitter, one is toward the sample and the other is for reference. The reference $\mathrm{THz}$ pulse was detected by a detection photoconductive antenna for the reference signal in order to monitor the jitter of the laser (i.e., amplitude and time-delay fluctuations). The other THz pulse was focused onto the ATR prism by another off-axis parabolic mirror with the incident angle set at $57^{\circ}$. The totally reflected $\mathrm{THz}$ pulse from the prism-sample interface was collimated and focused again by another series of off-axis parabolic mirrors onto the detection photoconductive antenna for the sample signal. This optical setup minimized system fluctuation and achieved a precise THz TD-ATR measurement of the time-domain $\mathrm{THz}$ pulses $E(t)$ as a function of the glucose concentration. After Fourier transforming the time-domain $\mathrm{THz}$ pulse $E(t)$ into the reflectance $R(\omega)$ and phase shift $\varphi(\omega)$ spectrum in the frequency-domain, the experimentally determined complex reflection coefficient was calculated: $\tilde{r}(\omega)=\sqrt{R(\omega)} e^{-i \varphi(\omega)}$. By substituting this experimentally determined $\tilde{r}(\omega)$ into the Fresnel equation, both the real and imaginary parts of the complex dielectric constants of the sample were determined. Details are described in Ref. 39.

At higher frequencies from 3 to $12 \mathrm{THz}$, FIR FT-ATR measurements were performed by a FARIS-1 s (Jasco, Ltd.). In this system, a ceramic heater and pyroelectric element (deuterated triglycine sulfate detector) were used as a light source and detector, respectively, and the silicon ATR prism $(300 \pm 0.1 \mathrm{~K})$ was set to the focal position of the FIR light at an incident angle of $45^{\circ}$. First, the measured unpolarized reflectance $R(\omega)$ was transformed into the $s$-polarized reflectance $R_{\mathrm{s}}(\omega)$ by the relationship $2 R(\omega) \equiv R_{\mathrm{s}}(\omega)+R_{\mathrm{p}}(\omega)=R_{\mathrm{S}}(\omega)+R_{\mathrm{s}}{ }^{2}(\omega) .{ }^{40}$ Note that this relationship holds only at $45^{\circ}$ incidence. $R_{\mathrm{S}}(\omega)$ was put into the Kramers-Kronig equation, Eq. (1), so as to calculate the $s$-polarized phase shift spectrum $\varphi_{\mathrm{s}}(\omega)$,

$$
\varphi_{\mathrm{s}}(\omega)=-\frac{2}{\pi} \int_{0}^{\omega_{\mathrm{u}}} \frac{\omega_{\mathrm{a}} \ln \sqrt{R_{\mathrm{s}}\left(\omega_{\mathrm{a}}\right)}}{\omega_{\mathrm{a}}^{2}-\omega^{2}} d \omega_{\mathrm{a}}+\varphi_{\infty},
$$

where, $\omega_{\mathrm{u}}$ is the upper integration limit and $\varphi_{\infty}$ represents the phase shift at $\omega=\omega_{\mathrm{u}}{ }^{40}$ In this case, the second term in the right-hand side $\left(\varphi_{\infty}\right)$ could not be experimentally derived from our measurements. To resolve this problem, we set $\varphi_{\infty}$ as the value that is best fitted to the THz TD-ATR measurement result between 2.8 and $3.0 \mathrm{THz}$, in order to smoothly connect the $\mathrm{THz}$ TD-ATR and FIR FT-ATR results with the deviations smaller than \pm 0.003 for both real and imaginary parts. ${ }^{41,42}$ Once the $\varphi_{\mathrm{s}}(\omega)$ was successfully determined, the experimental complex reflection coefficient $\tilde{r}_{\mathrm{s}}(\omega)=\sqrt{R_{\mathrm{s}}(\omega)} e^{-i \varphi_{\mathrm{s}}(\omega)}$ was substituted into the Fresnel equation to calculate the complex dielectric constant of the sample.

\section{Experimental errors}

In the commercial vector network analyzer system, the relative standard deviation $(\delta \tilde{\varepsilon}(\omega) / \tilde{\varepsilon}(\omega)$, where $\delta \tilde{\varepsilon}(\omega)$ represents the standard deviation of measurements) of water between 0.5 and $40 \mathrm{GHz}$ was less than $1.0 \%$ and $1.4 \%$ in the real and imaginary parts, respectively. These results are much better than a previous microwave dielectric spectroscopic study of saccharide solutions, ${ }^{23}$ which leads the reliability to our further analysis. Regarding the THz TD-ATR measurements $(0.25-3.0 \mathrm{THz})$, the relative standard deviation increased linearly with frequency; typical relative standard deviations in the real and imaginary parts of the complex dielectric constants of water were $0.2 \%$ and $0.3 \%$ at $0.5 \mathrm{THz}, 0.4 \%$ and $0.4 \%$ at $1.0 \mathrm{THz}, 1.0 \%$ and $0.8 \%$ at $2.0 \mathrm{THz}$, and $1.7 \%$ and $1.9 \%$ at $3.0 \mathrm{THz}$, respectively. The reason for the increase in the standard deviation at higher frequencies is attributed to a lower

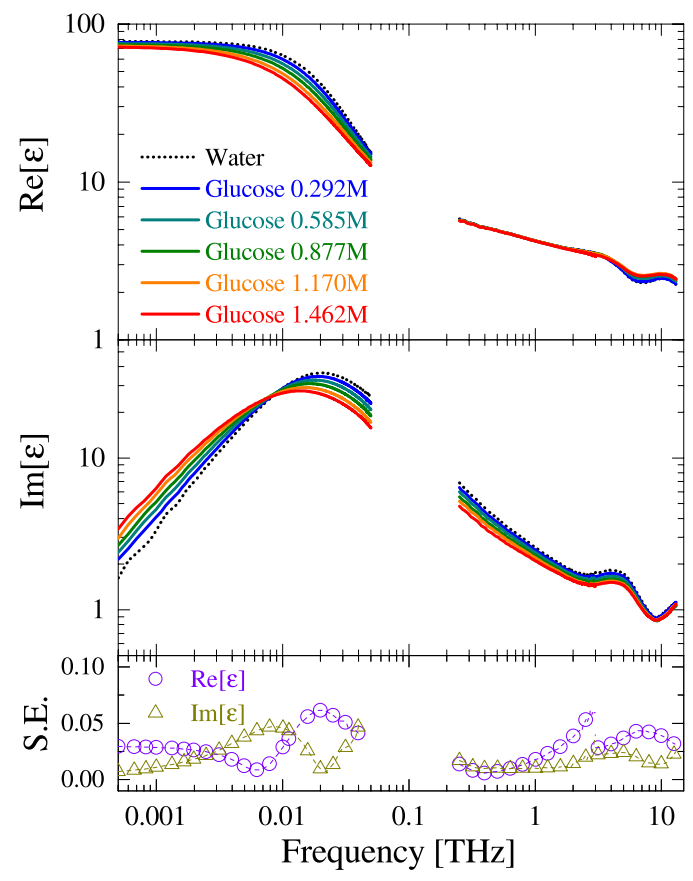

FIG. 1. The complex dielectric constants of liquid water and aqueous glucose solutions at $300 \mathrm{~K}$, between $0.5 \mathrm{GHz}$ and $12 \mathrm{THz}$. The bottom graph shows typical S.E. in the measurement systems. 
signal-to-noise ratio in the higher frequency region. For the THz TD-ATR spectrometer used, the highest signal-to-noise ratio was found around $0.5 \mathrm{THz}$, whereas that of $3.0 \mathrm{THz}$ was reduced by $30 \mathrm{~dB}$. Finally, the relative standard deviation of reflectance in the FIR FT-ATR measurements of water was $0.5 \%$ at $5 \mathrm{THz}$ and $0.9 \%$ at $10 \mathrm{THz}$. The frequency dependence of the standard errors (S.E.) of water in our measurement from $0.5 \mathrm{GHz}$ to $12 \mathrm{THz}$ is summarized at the bottom of Figure 1 .

\section{RESULTS AND DISCUSSIONS}

\section{A. Spectrum decomposition}

The measured complex dielectric constants of the distilled water and glucose aqueous solutions at $300 \mathrm{~K}$, from $0.5 \mathrm{GHz}$ to $12 \mathrm{THz}$, are shown in Figure 1. In the low frequency region, a large and broad dispersion is red-shifted accompanying the decrease in the peak height as glucose concentration increases. This tendency is in line with previously reported dielectric spectra between $1 \mathrm{MHz}$ and $20 \mathrm{GHz} .^{22}$ In the THz region, we observed a noticeable decrease in the imaginary part but the real part is almost unchanged, as was also seen in disaccharide sucrose solutions measured by Arikawa et al. ${ }^{30}$ However, no absorption peaks inherent in the glucose crystalline ${ }^{43-45}$ were evident in Figure 1, as would be expected since they originate from glucose-glucose intermolecular HBs and van der Waals forces, which should be markedly decreased in the aqueous phase. ${ }^{39}$

The frequency region $0.5 \mathrm{GHz}-12 \mathrm{THz}$ corresponds to a 0.01-318 ps time window, and thus, our experimental results reflect various molecular dynamics: from a solute tumbling motion to HB vibrations. However, the overlaps of these different molecular dynamics at the same frequency complicate the interpretation of the measured complex dielectric constants. In order to isolate the complex susceptibility of each molecular dynamic from the measured complex dielectric constant, the spectrum was decomposed into the Debye relaxation and Lorentz resonant functions. In case of biological macromolecular solutions, such as proteins, complex dielectric constants below $50 \mathrm{GHz}$ are known to be successfully decomposed into three different Debye relaxation components: $\beta$ relaxation (dipolar solute rotation or tumbling), $\delta$ relaxation (reorientation of hydrated water), and $\gamma$-relaxation (collective reorientation of the HB bulk water). ${ }^{46}$ In the case of saccharide solutions, additional relaxation modes assigned to structural relaxation of a saccharide molecule ${ }^{47}$ and dipolar fluctuation of the exocyclic hydroxylmethyl group $\mathrm{s}^{48,49}$ are present far below $0.5 \mathrm{GHz}$. Since their contribution to the complex dielectric constant between $0.5 \mathrm{GHz}$ and $12 \mathrm{THz}$ is supposed to be smaller than our measurement errors, the existence of these two relaxational modes is not accounted for in the present analysis.

For macromolecular (i.e., protein) aqueous solutions, the decomposition of the complex dielectric constant into $\beta, \delta$, and $\gamma$-relaxation modes has been frequently used, since $\beta$ relaxation is clearly separated from the other two relaxation modes. However, it is not an easy task to apply the same decomposition procedure to a saccharide (especially a monosaccharide) aqueous solution. This is because the time constants of the saccharide tumbling and orientational motion of hydrated water are very close to each other due to the smaller molar mass of the saccharides compared to that of proteins, resulting in an ambiguous boundary between $\beta$ and $\delta$ relaxations. ${ }^{50}$ Therefore, relaxation functions, such as the Cole-Cole, Cole-Davidson, or Havriliak-Negami, are conventionally used for saccharide solutions, instead of the Debye relaxation function. ${ }^{22-24}$ These functions describe the superposition of at least two different symmetric Debye functions as only one asymmetric relaxation component and thus are useful for the relatively simple treatment of complicated dynamics. However, previously reported dielectric spectroscopy studies in the $\mathrm{GHz}$ region ${ }^{22-24}$ suggest that although these functions can be successfully reproduced experimentally, detailed evaluation and discussion of the hydration state of saccharide solutes is problematic. In the present study, we decomposed the experimental results in the microwave regime into the Debye type $\beta, \delta$, and $\gamma$ relaxations without using such asymmetric functions, so that we could evaluate the hydration state more systematically. Furthermore, beyond the microwave region, the complex dielectric constants of distilled water and aqueous saccharide solutions up to $12 \mathrm{THz}$ were represented by adding another Debye function and two Lorentz resonant functions: ${ }^{41,42}$ fast relaxation (rotation of the non-HB bulk water that adrift from the HB network), intermolecular stretching vibration (hindered $\mathrm{HB}$ translational motion between $\mathrm{O} \cdots \mathrm{O}$ ), and libration (hindered $\mathrm{HB}$ rotational motion between $\mathrm{O} \cdots \mathrm{H}$ ). ${ }^{34,35}$

Thereby, the complex dielectric constants $\tilde{\varepsilon}(\omega)$ from $0.5 \mathrm{GHz}$ to $12 \mathrm{THz}$ can be expressed as a superposition of a total of six complex susceptibilities $\tilde{\chi}(\omega)$ as follows:

$\beta$ relaxation of the glucose solute $\tilde{\chi}_{\beta}(\omega)$,

$\delta$ relaxation of the hydrated water $\tilde{\chi}_{\delta}(\omega)$,

$\gamma$ relaxation of the HB bulk water $\tilde{\chi}_{\text {slow }}(\omega)$ (hereafter referred as "slow relaxation" in contrast to fast relaxation), fast relaxation of the non-HB bulk water $\tilde{\chi}_{\text {fast }}(\omega)$, intermolecular stretching vibration of water $\tilde{\chi}_{S}(\omega)$, and libration of water $\tilde{\chi}_{L}(\omega)$.

Accordingly, taking into account the high frequency limit of the real part $\varepsilon_{\infty}$, the function was modeled as follows:

$$
\begin{aligned}
\tilde{\varepsilon}(\omega)= & \tilde{\chi}_{\beta}(\omega)+\tilde{\chi}_{\delta}(\omega)+\tilde{\chi}_{\text {slow }}(\omega)+\tilde{\chi}_{\text {fast }}(\omega) \\
& +\tilde{\chi}_{\mathrm{S}}(\omega)+\tilde{\chi}_{\mathrm{L}}(\omega)+\varepsilon_{\infty} \\
= & \frac{\Delta \varepsilon_{\beta}}{1+i \omega \tau_{\beta}}+\frac{\Delta \varepsilon_{\delta}}{1+i \omega \tau_{\delta}}+\frac{\Delta \varepsilon_{\text {slow }}}{1+i \omega \tau_{\text {slow }}}+\frac{\Delta \varepsilon_{\text {fast }}}{1+i \omega \tau_{\text {fast }}} \\
& +\frac{\Delta V_{\mathrm{S}} \omega_{\mathrm{S}}^{2}}{\omega_{\mathrm{S}}^{2}-\omega^{2}+i \omega \gamma_{\mathrm{S}}}+\frac{\Delta V_{\mathrm{L}} \omega_{\mathrm{L}}^{2}}{\omega_{\mathrm{L}}^{2}-\omega^{2}+i \omega \gamma_{\mathrm{L}}}+\varepsilon_{\infty},
\end{aligned}
$$

where, $\Delta \varepsilon_{j}$ : relaxation strength, $\tau_{j}$ : relaxation time, $\Delta V_{k}$ : vibration strength, $\omega_{k}$ : resonant frequency, and $\gamma_{\mathrm{k}}$ : damping constant $(j=\beta, \delta$, slow and fast $/ k=\mathrm{S}$ and $\mathrm{L})$.

A nonlinear least-square fitting calculation based on the Levenberg-Marquardt algorithm ${ }^{51}$ was performed for the spectrum decomposition procedure until the chi-square is smaller than $10^{-9}$.

For a successful fit, $\tau_{\text {slow }}$ at $300 \mathrm{~K}$ was fixed to 7.93 ps for all the samples according to the critical slowing down formula of water. ${ }^{34,52}$ Although this formula originally holds in pure water, it has been found that $\tau_{\text {slow }}$ of bulk water is constant in biological solution at various concentrations. ${ }^{46}$ Therefore, 
we considered the fixation of $\tau_{\text {slow }}=7.93$ ps to be valid in the present case for bulk water, which is dynamically unperturbed by the solute..$^{41,42}$ Additionally, prior to the fitting procedure, $\tau_{\beta}$ was also fixed as per the commonly adopted theoretical expression (Stokes-Einstein-Debye relation), ${ }^{53}$

$$
\tau_{\beta}=\frac{4 \pi r^{3} \eta}{K_{\mathrm{B}} T},
$$

with a hydrodynamic radius $r=3.41 \AA$ for glucose ${ }^{54}$ with a macroscopic viscosity $\eta$ and thermal energy $K_{\mathrm{B}} T$. The viscosity $\eta$ of the aqueous samples was measured by an oscillating type viscosity meter SV-10 (A\&D, Ltd.). $\tau_{\beta}$ values used in the fitting procedure are shown in the inset in Figure 3, showing an exponential increment attributed solely to the viscosity $\eta$. The Stokes-Einstein-Debye relation assumes the rotational correlation time of a "single" particle in the medium. This assumption should be valid over the investigated concentration regime because of the far below monosaccharide percolation threshold. ${ }^{4}$ Since previous MD simulations have shown that the average number of glucose-glucose HB is, at most, 0.1 per solute molecule even in $29 \mathrm{wt}$. \% (or ca. $1.85 \mathrm{M}$ in molar concentration) aqueous solution, ${ }^{4}$ the glucose solute can be considered to be a single particle. The validation of this procedure has been also demonstrated by depolarized Raman scattering measurements, showing that the relaxation times $\tau_{\beta \text { (Raman) }}$ can be successfully reproduced by the Stokes-Einstein-Debye relation from low to markedly high concentration regimes. ${ }^{14}$ Please note, however, that the $\tau_{\beta}$ obtained in the present study is not equivalent to the $\tau_{\beta(\operatorname{Raman})}$ : the Stokes-Einstein-Debye type $\tau_{\beta}$ in the dielectric spectroscopy will be three times larger than the $\tau_{\beta(\text { Raman })}$, because the former reflects the first-rank $(l=1)$, whereas the Raman process reflects the second-rank $(l=2)$ susceptibility.

From Eq. (2), we fitted the parameters at $300 \mathrm{~K}$ for distilled water. They were $\Delta \varepsilon_{\text {slow }}=72.09 \pm 0.02, \Delta \varepsilon_{\text {fast }}$ $=1.98 \pm 0.02, \tau_{\text {fast }}=277 \pm 6 \mathrm{fs}, \Delta V_{\mathrm{S}}=1.23 \pm 0.03, \omega_{\mathrm{S}}=5.22$ $\pm 0.06 \mathrm{THz} / 2 \pi, \gamma_{\mathrm{S}}=5.43 \pm 0.18 \mathrm{THz} / 2 \pi, \Delta V_{\mathrm{L}}=0.50 \pm 0.04$, $\omega_{\mathrm{L}}=13.94 \pm 0.32 \mathrm{THz} / 2 \pi$, and $\gamma_{\mathrm{L}}=7.70 \pm 0.86 \mathrm{THz} / 2 \pi$. Each fitted result is in good agreement with previously reported values by Yada et al. ${ }^{34,35}$ The static dielectric constant of the distilled water at $300 \mathrm{~K}, \varepsilon_{\mathrm{s}}=\Delta \varepsilon_{\text {slow }}+\Delta \varepsilon_{\text {fast }}+\Delta V_{\mathrm{S}}$ $+\Delta V_{\mathrm{L}}+\varepsilon_{\infty}=77.69$, is also very close to the one obtained from an empirical formula (77.68). ${ }^{55}$ The broadband complex dielectric constant and its decomposed susceptibilities of the glucose $1.462 \mathrm{M}$ solution are shown in Figure 2. Our experimental result successfully reproduces the sum of the complex susceptibilities, for both real and imaginary parts. Furthermore, we substituted the best-fitted $\varepsilon_{\infty}$ into the Bruggemann effective medium theory, which describes the dielectric responses of mixed samples in the infrared or higher frequency regimes, ${ }^{30}$ in order to calculate the (high) frequency limit of the dielectric constant for glucose solutes, $\varepsilon_{\infty}^{(\mathrm{g})}$. In the present case, $\varepsilon_{\infty}^{(\mathrm{g})}$ should correspond approximately to the dielectric constant in the IR region, and the calculation results showed that $\varepsilon_{\infty}^{(\mathrm{g})} \approx 3.0$ was independent of concentration (data not shown). A reasonable value, since an $\varepsilon_{\infty}^{(\mathrm{g})} \approx 3.0$ falls between the dielectric constant of glucose crystals in the $\mathrm{THz}$ region (3.2) and the visible region (2.7) ${ }^{43}$ There was a general trend of the real part of the dielectric constant decreasing as

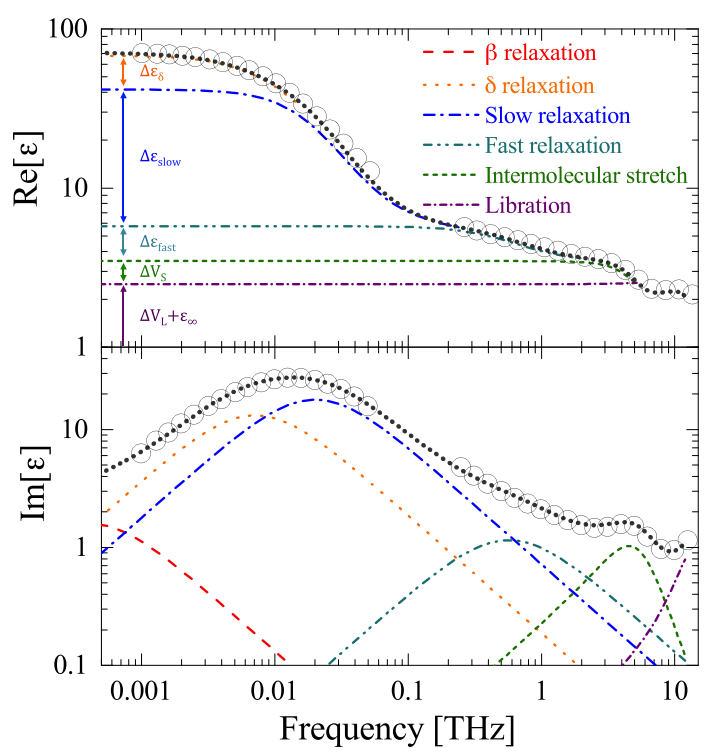

FIG. 2. The complex dielectric constant of $1.462 \mathrm{M}$ glucose solution decomposed into its constituents: (red) $\beta$ relaxation of the glucose solute, (orange) $\delta$ relaxation of the hydrated water, (blue) slow relaxation of the HB bulk water, (aqua) fast relaxation of the non-HB water, (green) intermolecular stretching vibration, and (purple) intermolecular libration.

frequency increased. These results confirm the validity of the fitting procedures for the glucose aqueous solutions.

\section{B. Dipole moment of the glucose solute}

The $\beta$ relaxation is an orientational process of a single solute molecule controlled by its own dipole, and thus, the relaxation strength $\Delta \varepsilon_{\beta}$ is related to the effective dipole moment of the solute in aqueous solution. ${ }^{46}$ Figure 3 shows the bestfit for $\Delta \varepsilon_{\beta}$ versus glucose concentration. Over the investigated concentration region, $\Delta \varepsilon_{\beta}$ linearly increases with concentration increase. But it should be noted that this first-order correlation is dominated by the fact that the number of glucose molecules (=dipole) linearly increases with concentration increase. In order to reveal the effective dipole moment of glucose $\mu_{\mathrm{eff}}$, the Onsager-Oncley model can be used, ${ }^{46}$

$$
\mu_{\mathrm{eff}}^{2}=\frac{2 \varepsilon_{0} K_{\mathrm{B}} T \Delta \varepsilon}{N_{\mathrm{A}} C}
$$

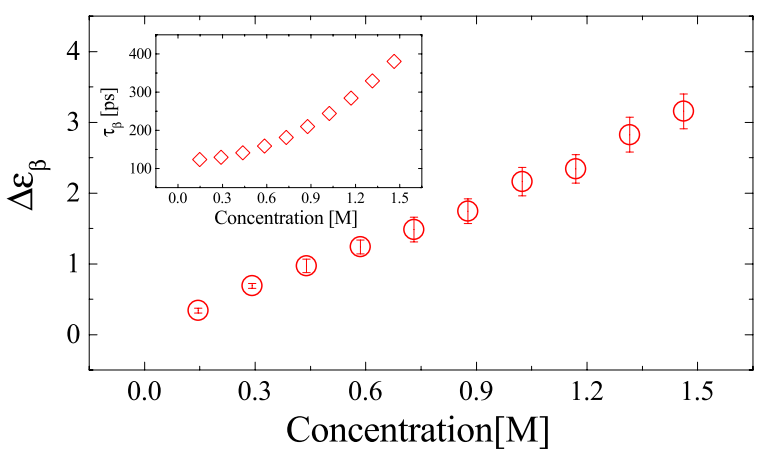

FIG. 3. Concentration dependence of the relaxation strength of the $\beta$ relaxation $\left(\Delta \varepsilon_{\beta}\right)$. The bars represent the analytical errors arisen from the leastsquare fitting procedure. The inset is the relaxation time $\left(\tau_{\beta}\right)$ calculated by Eq. (3). 


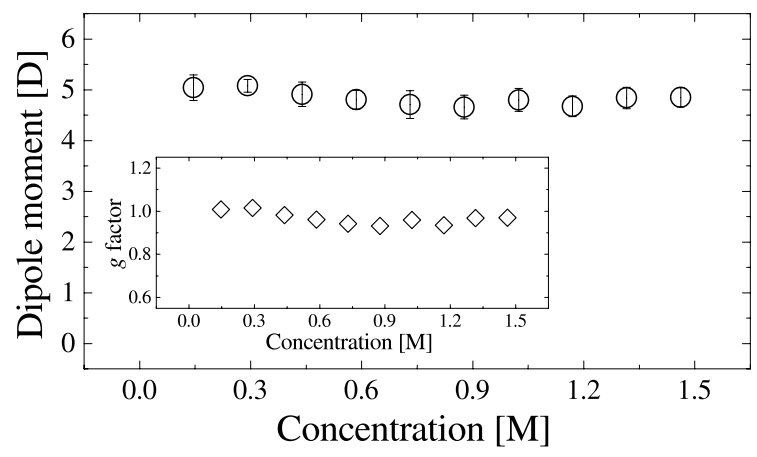

FIG. 4. The effective dipole moment $\mu_{\text {eff }}$ of glucose in aqueous solution at $300 \mathrm{~K}$, as a function of the concentration, derived from the dielectric strength of the $\beta$ relaxation. Inset shows the Kirkwood's $g$ factor.

where $\varepsilon_{0}$ is the vacuum permittivity, $N_{\mathrm{A}}$ is the Avogadro number, and $C$ is the molar concentration of glucose. As seen in Figure 4, the calculated effective dipole moment of glucose in aqueous solution is constant within the margin of analytical error. Seemingly, the present result $\left(\mu_{\mathrm{eff}}=4.9\right.$ $\pm 0.2 \mathrm{D}$ ) in the aqueous phase is much larger than that in the gas phase (between 2.4 and 4.2 depending on the adopted computational model and the anomeric conformation). ${ }^{56}$ The reason for this discrepancy of the dipole moment between the solution and gas phase is that the dipole moment is dependent on the surrounding environment, since the solute molecule in the aqueous phase is continuously affected by a reaction field (i.e., fluctuation of water dipole) even in the absence of an external field, leading to the larger effective dipole moment than the gas-phase molecule. ${ }^{46,57}$ In fact, our results are in agreement with previous MD simulation $(5.4 \pm 1.3 \mathrm{D})^{50}$ and theoretical study $(4.5 \mathrm{D})^{58}$ of glucose aqueous solution. Consequently, this confirms the appropriateness of the $\Delta \varepsilon_{\beta}$ value used in our analysis. It also indirectly affirms the appropriateness of the Stokes-Einstein-Debye relation to describe $\tau_{\beta}$ values in case of glucose aqueous solution.

In previous dielectric spectroscopy, it has been proposed that water and glucose cooperatively reorient, and thus, no glucose tumbling motion is evident in the complex dielectric constant of the glucose aqueous solution. ${ }^{22,24}$ By fixing $\tau_{\beta}$ by the Stokes-Einstein-Debye type relaxation time, however, we could isolate the reorientational relaxation of glucose molecules in the low $\mathrm{GHz}$ region. Unfortunately, a very small $\Delta \varepsilon_{\beta}$ due to a small dipole moment of glucose, typically 2 orders smaller than that of proteins, ${ }^{46}$ buries this relaxation mode in the $\delta$ relaxation of the hydrated water (see Figure 2). Nevertheless, our statement that the glucose relaxation component can be separated from water relaxation is consistent with recent depolarized scattering measurements, where solute relaxations are distinctly present because their spectrum does not represent a dipole moment but a polarizability anisotropy. ${ }^{14-18}$

As can be seen in Figure 4, the apparent dipole moment of glucose $\mu_{\text {eff }}$ exhibits little concentration dependence, which is in line with an earlier dielectric spectroscopic result. ${ }^{23}$ Once we introduce the Kirkwood correlation $g$ factor in order to describe the short-range dipole-dipole self-correlation with the relationship $\mu_{\text {eff }}^{2}=g \mu_{0}^{2}$ ( $\mu_{0}$ : dipole moment of isolated solutes), the dependence of the $g$ factor on the glucose concentration is shown in the inset of Figure 4. This $g$ factor is a parameter to account for molecular associations and correlation effects between the motions of solute and solvent molecules. ${ }^{59}$ Here, $\mu_{0}=5.0 \mathrm{D}$ is determined by extrapolation of the apparent dipole moment to zero concentration. ${ }^{46,60}$ As expected, the $g$ factor is unchanged over the entire glucose concentration region investigated, indicating that attractive or repulsive interactions between glucose molecules are negligibly small. This statement is consistent with a prerequisite of our analysis where the Stokes-Einstein-Debye equation is used, because the equation stands only when solute-solute interactions are negligible. Furthermore, the fact that the average number of glucose-glucose $\mathrm{HB}$ is at most 0.1 even at $1.85 \mathrm{M}$ as evaluated by previous MD simulation ${ }^{4}$ highlights the validity of our result.

\section{Glucose hydration number}

The $\delta$ relaxation mode is a so-called orientational motion of hydrated water. This will be contrasted with the slow relaxation $(\gamma$ relaxation) of the collective reorientation of the hydrogen-bonded bulk water. In this section, we will focus on the concentration dependence of the relaxation strength and relaxation time of these two relaxation modes to quantitatively discuss the hydration state. The relaxation strength of these relaxation modes directly represents the number of hydrated and bulk water molecules, and the relaxation time also indicates the hydration dynamics. The best-fit relations for the $\delta$ relaxation components $\left(\Delta \varepsilon_{\delta}\right.$ and $\left.\tau_{\delta}\right)$ and the slow relaxation component $\left(\Delta \varepsilon_{\text {slow }}\right)$ are shown in Figures 5 and 6, respectively. The slow relaxation strength decreases as glucose concentration increases with a drop-off more marked than would be expected if it was due just to the volume of water replaced by the solute molecules (represented as the broken line in Figure 6), suggesting that a fraction of the water molecules in the glucose solution are no longer behaving as bulk water. These water molecules are thought to have been bound up as

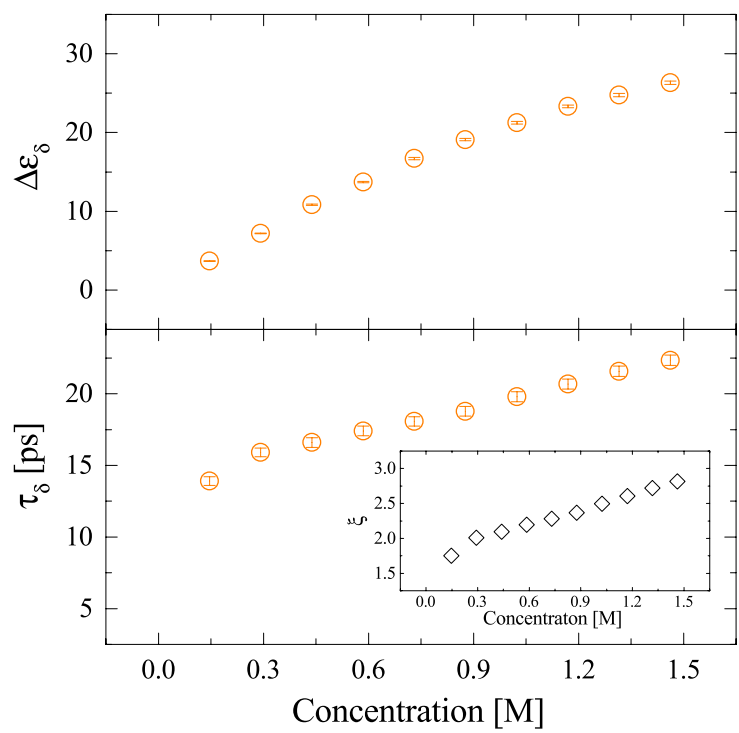

FIG. 5. (Top) Relaxation strength $\Delta \varepsilon_{\delta}$ and (bottom) relation time $\tau_{\delta}$ of the hydrated water versus concentration with analytical error bars. The inset shows the retardation factor $\xi=\tau_{\delta} / \tau_{\text {slow }}$. 


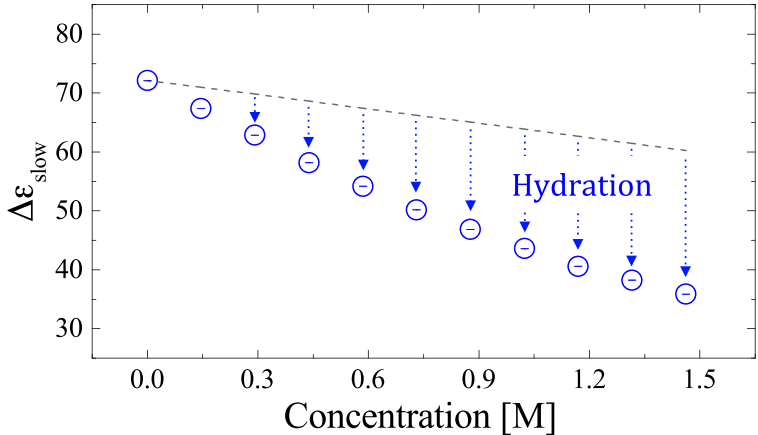

FIG. 6. Relation between the glucose concentration and $\Delta \varepsilon_{\text {slow. }}$. The upper broken line represents the ideal bulk water amplitude calculated from the analytical water concentration under the assumption that all water molecules in the solution are bulk water.

hydrated water, thus the large attenuation in $\Delta \varepsilon_{\text {slow }}$ (Figure 6) directly corresponds to an increase in the amount of hydrated water. ${ }^{46}$

In this article, we use two different ways to calculate the hydration number of glucose: one is the " $\delta$ hydration" and the other is the "whole hydration" number. The $\delta$ hydration number is calculated from the increase in the $\delta$ relaxation, while the whole hydration number is determined by the decrease in $\Delta \varepsilon_{\text {slow }}$. By comparing the " $\delta$ hydration" and "whole hydration," the existence of another potential relaxation component of hydrated water can be examined. We can assume that if the $\delta$ hydration number is identical to the whole hydration number, then all the hydrated water molecules are contributing to the $\delta$ relaxation component. On the other hand, if the whole hydration number exceeds the $\delta$ hydration number, another relaxation mode of hydrated water can be assumed to exist that is distinct from the $\delta$ relaxation, supposedly located at a much lower frequency. In fact, Cametti et al. ${ }^{46}$ noted two relaxation components of hydrated water in lysozyme aqueous solutions, peaking at around $0.1 \mathrm{GHz}$ and $2 \mathrm{GHz}$, assigned to tightly and loosely bound water, respectively. We will attempt to characterize the saccharide hydration mechanism in this way, since it has been vaguely understood so far. ${ }^{19,61}$

First, we determined the " $\delta$ hydration" number assuming that all the hydrated water molecules belong to the $\delta$ relaxation mode with a relaxation time of $\tau_{\delta}$. Recent ab initio MD simulations by Suzuki ${ }^{62}$ pointed out that the dipole moment of hydrated water differs by less than $2 \%$ from that of bulk water. In combination with the fact that the dielectric strength is proportional to the water content multiplied by square of its dipole moment, ${ }^{63}$ this provides assurance that the ratio $\Delta \varepsilon_{\delta} / \Delta \varepsilon_{\text {slow }}$ is equal to the fraction of hydrated to bulk water. ${ }^{46}$ Then, the molar concentration of the hydrated water $C_{\text {hyd }}(C)$ is given by

$$
\mathrm{C}_{\text {hyd }}(C)=\frac{\Delta \varepsilon_{\delta}(C)}{\Delta \varepsilon_{\delta}(C)+\Delta \varepsilon_{\text {slow }}(C)+\Delta \varepsilon_{\text {fast }}(C)} \frac{\rho_{\mathrm{w}}}{M_{\mathrm{w}}},
$$

where $\rho_{\mathrm{w}}$ : density of liquid water at $300 \mathrm{~K}$ and $M_{\mathrm{w}}$ : molar weight of water. In Eq. (5), the first term denotes the fraction of hydrated to total amount of water in solution. The " $\delta$ hydration" number per glucose solute $n_{\text {hyd }}^{(\delta)}$ is then calculated by the use of Eq. (6),

$$
n_{\text {hyd }}^{(\delta)}(C)=\frac{\mathrm{C}_{\text {hyd }}(C)}{C} .
$$

Second, the slow relaxation strength $\Delta \varepsilon_{\text {slow }}$ and the stoichiometric molar concentration of water in the aqueous solution are used to determine the "whole hydration" state. Within this framework, all the water molecules whose relaxation times are longer than bulk water $\left(\tau_{\text {slow }}=7.93 \mathrm{ps}\right)$ are regarded as hydrated water. From the slow relaxation component, the apparent bulk water concentration $C_{\text {bulk }}(C)$ was determined by $^{31,41,42}$

$$
\mathrm{C}_{\text {bulk }}(C)=\frac{\Delta \varepsilon_{\text {slow }}(C)+\Delta \varepsilon_{\text {fast }}(C)}{\Delta \varepsilon_{\text {slow }}(0)+\Delta \varepsilon_{\text {fast }}(0)} \frac{\rho_{\mathrm{w}}}{M_{\mathrm{w}}},
$$

where $\Delta \varepsilon_{\text {slow }}(0)$ was the slow relaxation strength of the distilled water. Together with the stoichiometric water molar concentration $C_{\text {water }}(C)$, which can be derived from the mol weight and the mass of water, the molar concentration of "whole hydrated" water is represented as $C_{\text {water }}(C)-C_{\text {bulk }}(C)$. Then, the "whole hydration" number per a solute $n_{\text {hyd }}^{(\text {w) }}$ is derived from the following equation: ${ }^{46}$

$$
n_{\text {hyd }}^{(\mathrm{w})}(C)=\frac{\mathrm{C}_{\mathrm{water}}(C)-\mathrm{C}_{\text {bulk }}(C)}{C} .
$$

Both hydration numbers, $n_{\text {hyd }}^{(\delta)}$ and $n_{\text {hyd }}^{(\mathrm{w})}$, are summarized in Figure 7 as a function of glucose concentration. Though the " $\delta$ hydration" number is only 3\%-4\% larger than the "whole hydration" number, $n_{\text {hyd }}^{(\delta)}$ is assumed to be equal to $n_{\text {hyd }}^{(\text {w) }}$ within the analytical errors. Hence, we can assert that all the hydrated water molecules in the glucose aqueous solutions contribute to the $\delta$ relaxation, and a further relaxation component with a significantly longer relaxation time does not exist. In particular, as shown in Figure 5, the relaxation time of the hydrated water molecules fell between 14 and 23 ps, a time slower than that of bulk water at $300 \mathrm{~K}, \tau_{\text {slow }}=7.93$ ps. This result can be interpreted as the retarded reorientational dynamics of hydrated water relative to bulk water. The extent of retardation is often represented as a retardation factor $\xi$, defined as $\xi=\tau_{\delta} / \tau_{\text {slow }}$. The concentration dependent retardation factor is displayed in the inset of Figure 5. We found a linear correlation of the retardation factor $\xi$ with increasing glucose concentration (from $0.146 \mathrm{M}$ to $1.462 \mathrm{M}$ ), where $\xi$ ranges from 1.7 to 2.8 in line with a previous dielectric spectroscopy report $(2.6)^{64}$

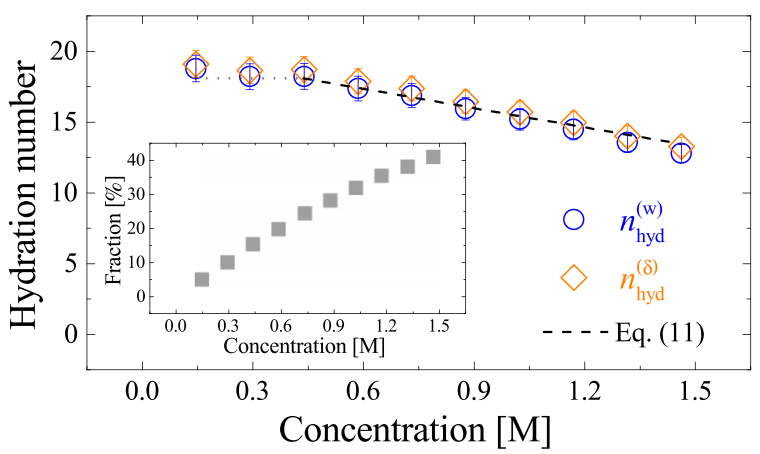

FIG. 7. $\delta$ hydration number $\left(n_{\text {hyd }}^{(\delta)}\right)$ and whole hydration number $\left(n_{\text {hyd }}^{(\mathrm{w})}\right)$ per a glucose solute at different concentrations. The gray broken line is an "analytical" value assuming the overlaps of the hydration layers, as calculated by Eq. (11). The inset exhibits the ratio of hydrated water to total water. 
and MD simulations (2.5). ${ }^{6}$ On the other hand, Lupi et al. ${ }^{17,18}$ have reported a larger retardation factor, $\xi=5-6$, for both mono- and disaccharides using depolarized light scattering. This discrepancy may arise from the different molecular dynamics probed. That is, depolarized light scattering probes the anisotropic relaxation of water translation, while the present dielectric spectroscopy measures the rotational relaxation: in other words, depolarized light scattering spectroscopy measures the density fluctuation in the hydration shell, whereas orientational dynamics of hydrated water can be probed by dielectric spectroscopy. ${ }^{65}$ As an earlier MD simulation has pointed out, ${ }^{6}$ the translational and rotational dynamics of water in the hydration shell are decoupled, reasonably explaining the difference in $\xi$ between our and Lupi et al. results. ${ }^{17,18}$ In this context, interestingly, the fact of a smaller retardation in the orientational than the translational direction evokes an image of more restricted collective relaxation dynamics in the translational direction in the saccharide hydration layer. Furthermore, a retardation factor of up to 2.8 in this study does not have to call on the existence of dynamically immobilized or icelike hydrated water molecules in the hydration shell. Our result differs from the protein hydration analogy, where strongly and moderately hydrated water exhibits two separated relaxational dispersions around $0.1 \mathrm{GHz}(\xi \approx 200)$ and $2 \mathrm{GHz}(\xi \approx 10)$, respectively. ${ }^{46}$ The greater extent of retardation for protein (at least $\xi \approx 10$ ) than glucose solutes may be attributed to a wider variety of chemical groups in the proteins, which have a larger molecular weight and various polar and apolar groups in close proximity. It has been speculated that this will simultaneously and effectively restrain water motions. ${ }^{6}$ More specifically, the presence of uniformly distributed $\mathrm{OH}$ groups on the glucose surface can be hypothesized to facilitate the accommodation of solute molecules in the water HB network, due to the small perturbation on the surrounding water's structure. ${ }^{54}$

The hydration number of the present study at $C \rightarrow 0$, $n_{\text {hyd }}^{0}=21.0 \pm 0.7$ per a glucose solute, is much larger than that determined from ultrasound ( 3.5 and 8.4) $)^{66,67}$ and calorimetric studies (5.0). ${ }^{68}$ This discrepancy can be attributed to a different definition of hydrated water. Indeed, they measured a "static" aspect of the hydrated water, focusing on the compressibility or melting point of water molecules, while the criterion for distinction between the bulk and hydrated water lies in its dynamical aspects in our analysis. Also, the $n_{\text {hyd }}^{0}=21.0 \pm 0.7$ is slightly larger than the recent depolarized light scattering measurements (14-16), ${ }^{17,18}$ probably owing to the distinctive molecular dynamics probed by that technique, as described above. Recently, Havenith et al. ${ }^{32,33}$ used $\mathrm{THz}$ vibrational spectroscopy around $2.5 \mathrm{THz}$ to measure the saccharide hydration state, based on the dipole fluctuation at a sub-picosecond time scale (corresponding to $2.5 \mathrm{THz}$ ). They decomposed the absorption spectrum $\alpha(\omega)$ into the solute, bulk water, and hydrated water by $\alpha(\omega)=\alpha_{\text {sol }}(\omega) \Phi_{\text {sol }}+\alpha_{\text {bulk }}(\omega) \Phi_{\text {bulk }}$ $+\alpha_{\text {hyd }}(\omega) \Phi_{\text {hyd }}$, by fixing $\alpha_{\text {sol }}(\omega), \Phi_{\text {sol }}$ and $\alpha_{\text {bulk }}(\omega)$, where $\Phi$ represents the volume fraction of each component. From the best-fitted $\Phi_{\text {hyd }}$, it was concluded that up to 50 water molecules could be perturbed by a glucose solute, ${ }^{69}$ which is noticeably larger than our result $\left(n_{\text {hyd }}^{0} \approx 21\right)$. Considering the first hydration layer of glucose can accommodate a maximum of approximately 35 water molecules, ${ }^{54}$ the authors speculated that the hydration effect of glucose reaches the second hydration shell (described as a "long-range" hydration effect). ${ }^{32,33}$ This might be probably because the dipolar dynamics at the sub-picosecond vibrational motions is more sensitive to minor dynamical perturbation of hydrated water than picosecond reorientational motion. However, their analytical process has been criticized by Winther et al. ${ }^{19}$ pointing out the result of Havenith et al ${ }^{32,33}$ implies that the second hydration layers differ less than $1 \%$ from bulk water in its $\mathrm{THz}$ absorption, which is too small to have significant consequences. Additionally, it should be also highlighted that they ignored any relaxational dynamics of water that actually contribute to the absorption spectrum around $2.5 \mathrm{THz}$ (see Figure 2(b)). In particular, the fast relaxation mode of bulk water, where the imaginary part encompasses about $30 \%$ at $2.5 \mathrm{THz}$, tends to increase as concentration increases (details will be shown in Sec. III E). This means that frequency dispersion of $\alpha_{\text {bulk }}(\omega)$ changes slightly as a function of concentration. Thus, their postulation that the frequency dispersion of $\alpha_{\text {bulk }}(\omega)$ is independent of concentration (i.e., the fixation of $\alpha_{\text {bulk }}(\omega)$ ) would result in overestimation of $\Phi_{\text {hyd }}$. Therefore, a little more refinement of their analysis will yield a more holistic picture of hydration, in combination with other experimental results.

Classical and $a b$ initio MD simulations have determined that the total average number of glucose-water HBs is about $10-11,,^{20,62,70}$ depending on anomeric structure and concentration, which can be defined as directly hydrated water. Additionally, if van der Waals forces are taken into account, the total hydration number amounts to $19-20,{ }^{20}$ in good agreement with the present result, $n_{\text {hyd }}^{0}=21.0 \pm 0.7$. Since the van der Waals forces are much weaker than HBs, we can interpret the breakdown of $n_{\text {hyd }}^{0}=21.0$ as 11 hydrated water molecules directly bound to the glucose polar groups via donor- or acceptor-HBs with a relatively large retardation, with the remainder of the hydrated water being weakly or indirectly perturbed by the glucose solutes and having relatively similar relaxation times with bulk water. On the basis of this concept, the concentration dependence of the hydration state is discussed in Sec. III D.

\section{Concentration-dependent hydration dynamics}

As shown in Figures 5 and 7, the reorientational relaxation time $\left(\tau_{\delta}\right)$ and the hydration number $\left(n_{\text {hyd }}\right)$ exhibit an almost linear correlation with glucose concentration.

To gain insight into this issue, we first examined the calculated distance between nearest neighbor solutes. If the glucose molecules, with a hydrodynamic molecular radius $r_{\mathrm{g}}=3.41 \AA$, are placed in a uniformly expanded cubic lattice, and given the volume fraction of the glucose solute $\Phi_{\mathrm{g}}$, the center-tocenter distance $D$ between nearest glucose molecules can be calculated as follows: ${ }^{19,71}$

$$
D=\sqrt[3]{\frac{4 \pi r_{\mathrm{g}}{ }^{3}}{3} \frac{1}{\Phi_{\mathrm{g}}}}
$$

The half-separation distance between the surfaces of the glucose molecules is calculated by $d=D / 2-r_{\mathrm{g}}$, as illustrated in Figure 8. As a first approximation, the thickness of the 


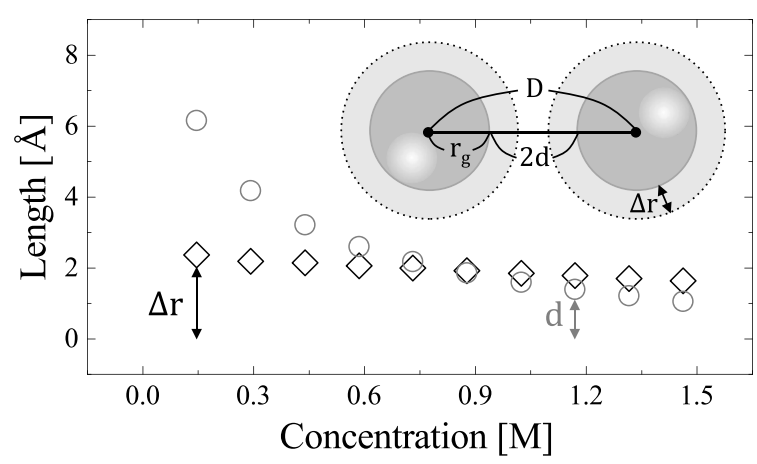

FIG. 8. Concentration-dependent glucose separation distance $(2 d)$ with the thickness of the hydration shell $(\Delta r)$. The inset shows a schematic illustration of the relationship of $d$ and $\Delta r$ with the center-to-center distance $D$ and the solute radius $r_{\mathrm{g}}$.

hydration shell $\Delta r$ is geometrically derived from Eq. (10), assuming the hydration layer is uniformly distributed around the spherical glucose molecule with radius $r_{\mathrm{g}}$,

$$
\frac{4 \pi\left(r_{\mathrm{g}}+\Delta r\right)^{3}}{3}-\frac{4 \pi r_{\mathrm{g}}{ }^{3}}{3}=n_{\mathrm{hyd}} \frac{4 \pi r_{\mathrm{w}}{ }^{3}}{3},
$$

where $r_{\mathrm{w}}$ is the hydrodynamic radius of water. In Eq. (10), the left-hand side represents the geometrical volume of the hydration shell and the right-hand side is the volume of water molecules packed in the hydration shell. The concentrationdependent $d$ and $\Delta r$ are compared in Figure 8. While the halfseparation distance $d$ exponentially decreased with increasing concentration due to a solute crowding effect, the thickness of the hydration shell $\Delta r$ more slowly decreases from 2.4 to 1.6 A. As a consequence, we find $d$ approaches $\Delta r$ at $0.6 \mathrm{M}$, suggesting, from a geometrical point of view, the hydration shells start to overlap around this concentration. From a RDF around glucose hydroxyl oxygen atoms, the thickness of the first hydration shell is estimated to be $3 \AA^{6}{ }^{6}$ which is greater than the determined $\Delta r$ in our study. The reason for the thinner thickness of hydration shell in this study $(\Delta r)$ is that the hydration shell is defined as being uniformly distributed around a glucose molecule, while in the case of RDFs, the localized distribution of the hydration shell around a specific atom is considered.

To elucidate the concentration dependence of $n_{\text {hyd }}$, an analytical model describing the overlapping of the hydration shells of close-to-contact solute molecules was adopted, ${ }^{54}$ as explained below. This analytical model is a simple geometric one that quantifies the number of water molecules in the hydration shell shared by the two closest solute molecules. According to Ref. 54, the concentration-dependent hydration number $n_{\text {hyd }}(C)$ is described as

$$
n_{\text {hyd }}(C)=n_{\text {hyd }}^{0}-\varrho_{\mathrm{w}} \varrho_{\mathrm{s}} \int_{2 r_{\mathrm{g}}}^{2\left(r_{\mathrm{g}}+\Delta r\right)} V(C) 4 \pi R^{2} d R,
$$

where $\varrho_{w(s)}$ is the number density of water (solute), $V(C)$ is the volume shared by the two solute molecules, and $R$ is the distance from the center of the solute molecule. In Eq. (11), $\varrho_{\mathrm{w}}$ is fixed at $0.0334 / \AA^{3}$, assuming negligible changes in the density of water around the solute. By substituting $\Delta r$ (in Figure 8) into Eq. (11), the "analytical" hydration number that takes account of the overlap effects of the hydration shells was determined (Figure 7, gray broken line). It is noteworthy that the "analytical" hydration number, as calculated by Eq. (11), decreased at almost the same rate as those in our experimental results, especially above $0.45 \mathrm{M}$, suggesting the decrease in $n_{\text {hyd }}(C)$ is sufficient to fully account for the overlap of the hydration shells. From this result, it is confirmed that glucose-glucose interactions and aggregation can be neglected, similar to that in Sec. III B, because solute aggregation or pseudo-clusterization further decreases the effective volume of hydrated water. On the other hand, it is shown that the slope of $n_{\text {hyd }}(C)$ becomes smaller below $0.45 \mathrm{M}$, indicating that the overlap of the hydration shells is not significant at these dilute glucose concentrations.

As mentioned in Sec. III C, 10 or 11 water molecules are estimated to directly form HBs with the glucose solute, ${ }^{20,62,70}$ typically each glucose $\mathrm{OH}$ forming one weaker acceptor and one stronger donor HB. ${ }^{62}$ The hydration number at the highest concentration in this study $(1.462 \mathrm{M}), n_{\mathrm{hyd}} \approx 13.5$, is slightly greater than that value, and thus, it is expected that the hydrated water indirectly perturbed by glucose polar groups or loosely bound to a glucose solute by van der Waals forces will be preferentially shared between neighboring glucose molecules. This is because these "weakly" hydrated water molecules are located at a greater distance than the "strongly" hydrated water that is directly hydrogen-bonded with the glucose $\mathrm{OH}$ groups. On the basis of this consideration, the inflection point of $n_{\text {hyd }}(C)$ around $0.45 \mathrm{M}$ is the point where the "weakly" hydrated water is likely to be shared with the proximal glucose, reducing the average hydration number per solute (see Figure 7). Since such weakly hydrated water is supposed to have a smaller $\tau_{\delta}$ than the strongly hydrated one directly bonded with glucose $\mathrm{OH}$ groups, the decrease in the number of weakly hydrated water per solute may account for the slowing down of the average reorientational motion $\left(\tau_{\delta}\right)$. In summary, the concentration-dependent tendency, as witnessed in Figures 5 and 7, is explained by the fact that the population of directly hydrated water remains almost unchanged, while the loosely or indirectly hydrated water, with the relatively small $\tau_{\delta}$, decreases as glucose concentration increases.

\section{E. Destructuring effect on the HB network}

Saccharides, with abundant polar $\mathrm{OH}$ groups and high electronegativity, ${ }^{13}$ are known to stabilize water-water HBs with a high enthalpy into saccharide-water HBs with a low enthalpy state. Seemingly in stark contrast to this widely accepted "structure-making" aspect, it has been computationally and experimentally found that the water HB network is somehow destructured in the presence of saccharides. ${ }^{6,8,10}$ This "destructuring effect" on the HB network by saccharides has been explained as follows: while the reorientational dynamics of water are stabilized (or "structured") by saccharide-water HBs, the water-water HBs are distorted in the vicinity of the hydrated saccharide complex. Such a destructured HB network of water is thought to hinder dehydration and freezing in plants or microorganisms. ${ }^{10}$ Branca et al..${ }^{8,9}$ intensively investigated this topic using, mainly, Raman scattering and focused on the anharmonic intramolecular $\mathrm{OH}$ stretching vibration around 
$100 \mathrm{THz}$, whose absorption peak can be disentangled into two $\mathrm{OH}$ stretching components; one is an orderly bonded water in the tetrahedral coordination and the other is disorderly bonded water. They concluded that the presence of saccharides tends to decrease the fraction of the former and increase the fraction of the latter. Lerbret et al. ${ }^{7,10}$ further asserted that the presence of saccharides increases the low HB number species (water that forms 0 or $1 \mathrm{HB}$ ). Raman scattering studies do, however, suffer from a masking effect where both the water and the saccharides' $\mathrm{OH}$ groups contribute to the $\mathrm{OH}$ stretch absorption band. To shed some more light on the mechanisms and characteristics of saccharides destructuring effect, we quantitatively examine the extent of non-HB water released from the water $\mathrm{HB}$ network and the structural distortion of the dynamical tetrahedral coordination of the water HB network by the saccharide. This is based on the dielectric responses obtained from the $\mathrm{THz}$ to FIR regions. Unlike the $\mathrm{OH}$ stretching around $100 \mathrm{THz}$, these frequency regions reflect the relaxation and vibration of the water molecules themselves without the direct interference from saccharides, since any relaxational and vibrational motions originating from the saccharides are absent or negligibly small. ${ }^{30,31}$

First, we experimentally demonstrated the existence and quantified the non-HB bulk water released from the HB network as a result of the destructuring effect of glucose. This was achieved by noting the fast relaxation mode of water, which is assigned to the non-HB bulk water rotational motion isolated from the HB network. The peak of this fast relaxation dispersion with a sub-picosecond relaxation time is located in the gap between the absorption peaks for slow relaxation and intermolecular stretching, as seen in Figure 2, and is subsumed by the tails of these two larger absorption modes. However, with precise broadband spectroscopic analysis of the slow relaxation and intermolecular stretching vibration modes, we can accurately extract the fast relaxation component. ${ }^{34,35}$ The best-fit correlations for $\Delta \varepsilon_{\text {fast }}$ and $\tau_{\text {fast }}$ with glucose concentration are shown in Figure 9. Surprisingly, the fast relaxation strength $\Delta \varepsilon_{\text {fast }}$ increased linearly, even though the molar concentration of bulk water is reduced with increasing glucose concentration. The almost constant $\tau_{\text {fast }}$ reflects the relatively small influence of the solute on the rotational motions of the non-HB water itself. These results indicate that the glucose solutes are instrumental in creating more non-HB water, arguably via the $\mathrm{HB}$ destructuring effect. To quantitatively estimate

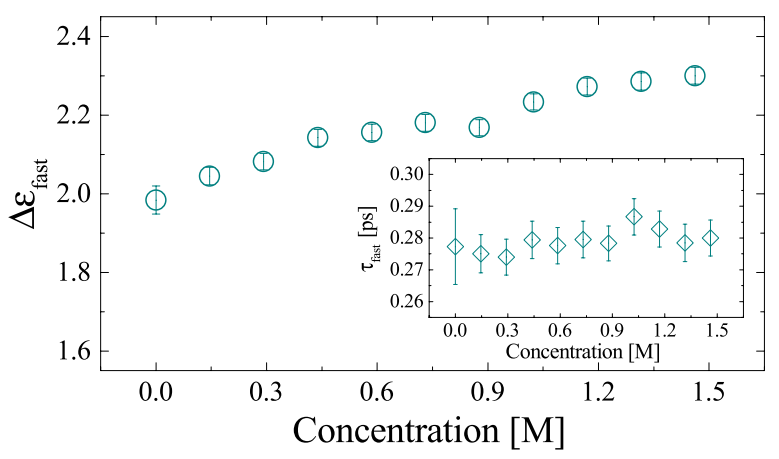

FIG. 9. Concentration dependence of the relaxation strength $\left(\Delta \varepsilon_{\text {fast }}\right)$ with analytical error bars. The inset is the fast relaxation time $\tau_{\text {fast }}$ versus glucose concentration.

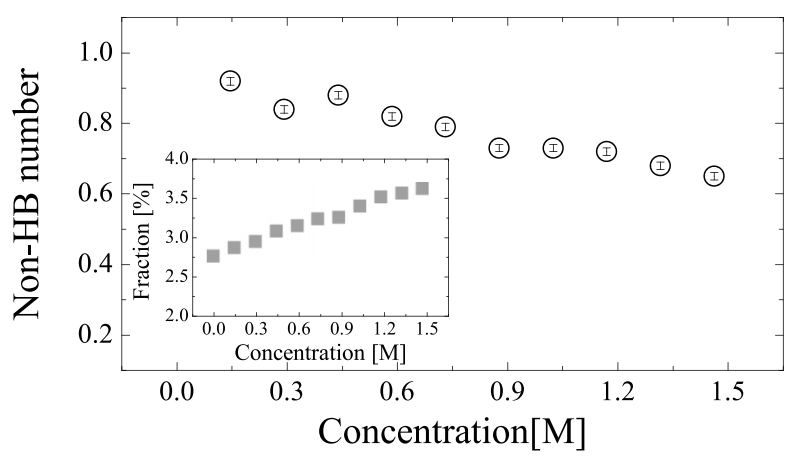

FIG. 10. The number of the non-HB water per a solute induced by a glucose solute $\left(n_{\text {non-HB }}\right)$ as a function of glucose concentration. The inset shows the fraction of non-HB water to bulk water.

this effect, the fraction of the non-HB water to total amount of water (including hydrated and bulk) in the glucose aqueous solutions, $\sigma_{\text {non-HB }}$, are calculated by Eq. (12),

$$
\sigma_{\text {non-HB }}(C)=\frac{\Delta \varepsilon_{\text {fast }}(C)}{\Delta \varepsilon_{\delta}(C)+\Delta \varepsilon_{\text {slow }}(C)+\Delta \varepsilon_{\text {fast }}(C)} .
$$

The fraction of non-HB to total amount of water increased as a function of concentration, from $2.77 \% \pm 0.03 \%$ for the distilled water to $3.61 \% \pm 0.03 \%$ for the $1.462 \mathrm{M}$ glucose solution (see inset, Fig. 10). This is consistent with the heat capacity measurements of Batchelor et al. ${ }^{72}$ who showed a negative partial molar heat capacity for glucose solutions, originating from more dense and less structured water species. In fact, in the transient dense-phase water regions, such "interstitial water," at the expense of any HBs with its neighbors, forms closely packed arrangement. ${ }^{73,74}$

The molar concentration of the non-HB water $\left(\mathrm{C}_{\text {non-HB }}\right)$ can be further delineated by ${ }^{41,42}$

$$
\mathrm{C}_{\text {non-HB }}(C)=\sigma_{\text {non-HB }}(C) \mathrm{C}_{\text {water }}(C) .
$$

Therefore, the number of glucose-induced non-HB water per a solute $\left(n_{\text {non-НB }}\right)$ can be derived by Eq. (14), ${ }^{31,41,42}$

$$
n_{\text {non-HB }}(C)=\frac{C_{\text {non-HB }}(C)}{C}-\frac{\Delta \varepsilon_{\text {fast }}(0)}{\Delta \varepsilon_{\text {slow }}(0)+\Delta \varepsilon_{\text {fast }}(0)} \frac{C_{\text {bulk }}(C)}{C} .
$$

The first term is the total number of the non-HB water assigned to a glucose molecule, and the second term denotes the unperturbed non-HB water in the background, in other words, the non-HB water that is so far away from the solute molecule that it is uninfluenced by the solute. Accordingly, the subtraction of the second term from the first term provides an estimate of the number of non-HB water in the vicinity of the solute, which directly arises from the destructuring effect. As seen in Figure $10, n_{\text {non-HB }}$ is positive at each concentration, confirming the destructuring effect on the water HB network. Our result is consistent with a previous Monte Carlo simulation ${ }^{74}$ and a state-of-the-art neutron scattering experiment, ${ }^{75}$ which stated the number of water monomer (non-HB water) increased in the first shell of saccharides. Nevertheless, $n_{\text {non-HB }}$ decreased by approximately $30 \%$ as concentration increased from $0.146 \mathrm{M}$ to $1.462 \mathrm{M}$. Since this tendency is in good agreement with the concentration dependent change of $\Delta r$, the thickness of the hydration shell (Figure 8), it is assumed that the decrease in 


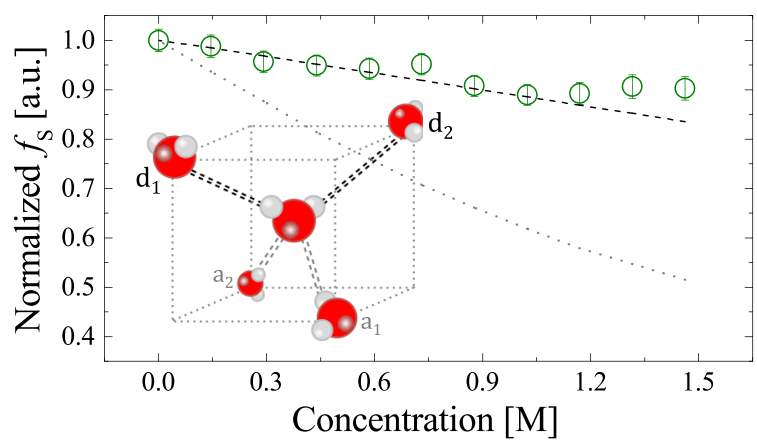

FIG. 11. Concentration dependent oscillation strength of the intermolecular stretching mode $\left(f_{\mathrm{S}}\right)$, where the bars indicate the errors arisen from the least-square fitting procedure. The upper broken line assumes all the water molecules in the aqueous solution contribute to this vibration mode, while the lower dot line is an analytical value under the assumption that only bulk water contributes to $\Delta \mathrm{V}_{\mathrm{S}}$. The inset is an ideal (ice-like) tetrahedral environment with two donor- and acceptor-HBs.

$n_{\text {non-HB }}$ is closely associated with the thickness of the hydration shells.

Next, the structural distortion of the dynamical tetrahedral HB network of water will be discussed, from the perspective of the intermolecular stretching vibration mode located around $5 \mathrm{THz}$. This intermolecular stretch is assigned to an intermolecular $\mathrm{O} \cdots \mathrm{O}$ translational motion in tetrahedrally coordinated structures, in particular the hindered $\mathrm{O} \cdots \mathrm{O}$ translation within $\mathrm{d} 1 \leftrightarrow \mathrm{d} 2$ and a1 $\leftrightarrow$ a2 2 sites (see inset, Fig. 11). ${ }^{76,77}$ Thus, this translational motion of water is originally Raman active but becomes IR active due to the intermolecular charge transfer accompanied by the water displacement. ${ }^{34,77-79}$ Experiments $^{80,81}$ and MD simulations ${ }^{82,83}$ have shown that, interestingly, fully hydrogen-bonded structures with a tetrahedral coordination are important key to activate this vibration mode, and the oscillation strength becomes significantly weaker in an incomplete tetrahedral structure like a supercritical environment. ${ }^{84}$ In this respect, this intermolecular vibration mode directly reflects the $\mathrm{HB}$ environment, especially the tetrahedrally coordinated HB environment. ${ }^{41,42}$ Based on this vibration mode, Yada et al. ${ }^{34}$ measured the $\mathrm{THz}$ spectrum of water and its isotopes $\left(\mathrm{H}_{2} \mathrm{O}, \mathrm{D}_{2} \mathrm{O}\right.$, and $\left.\mathrm{H}_{2}{ }^{18} \mathrm{O}\right)$ and ranked the tetrahedral disorder of these as $\mathrm{H}_{2} \mathrm{O}>\mathrm{D}_{2} \mathrm{O}>\mathrm{H}_{2}{ }^{18} \mathrm{O}$. On the basis of this line of research, we investigated the tetrahedrality of water around a solute, estimating to what degree the water $\mathrm{HB}$ structure is distorted in the glucose aqueous solutions.

To begin with, we calculated the oscillation strength $f_{\mathrm{S}}$ according to $^{34}$

$$
f_{\mathrm{S}} \propto \int \omega \operatorname{Im}\left[\tilde{\chi}_{\mathrm{S}}(\omega)\right] d \omega,
$$

where $\operatorname{Im}\left[\tilde{\chi}_{\mathrm{S}}(\omega)\right]$ is the imaginary part of the complex susceptibility of the intermolecular stretching vibration. The concentration dependence of $f_{\mathrm{S}}$ (Fig. 11), where both the HB bulk and hydrated water are assumed to contribute to this vibration (upper broken line) or where it is assumed that only the HB bulk water is responsible for $\tilde{\chi}_{\mathrm{S}}(\omega)$ (lower dotted line)-but not the hydrated water-was simulated. The results clearly fit the former assumption (upper broken line), indicating that $\tilde{\chi}_{\mathrm{S}}(\omega)$ best reflects the HB dynamics of the hydrated water, as well as the HB bulk water molecules. In a framework where the

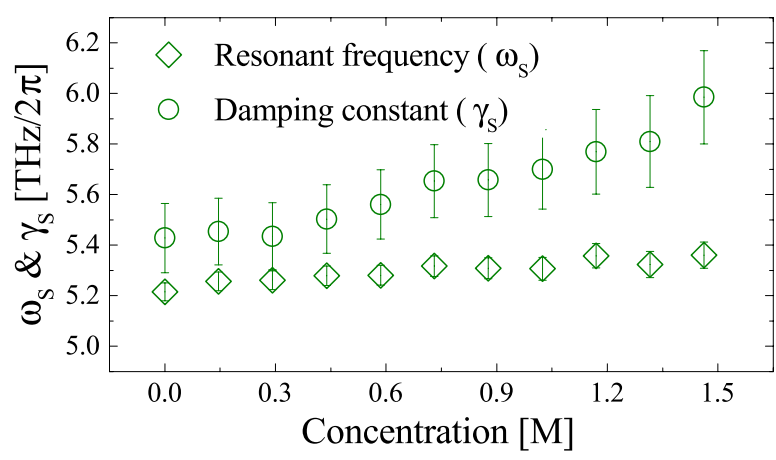

FIG. 12. (Diamond) Resonant frequency $\omega_{\mathrm{S}}$ and (circle) damping constant $\gamma_{\mathrm{S}}$ of glucose aqueous solution at different concentrations with error bars. A large increase in the damping constant reflects inhomogeneous distribution of the HB network.

$\tilde{\chi}_{\mathrm{S}}(\omega)$ mode is active under a tetrahedral architecture, ${ }^{78-83}$ this result implies that the hydrated water still remains tetrahedrallike in structure.

As seen in Fig. 12, we found the resonant frequency $\left(\omega_{\mathrm{S}}\right)$ and damping constant $\left(\gamma_{\mathrm{S}}\right)$ increased by $3 \%$ and $10 \%$, respectively, as glucose concentration increased to the maximum concentration investigated $(1.462 \mathrm{M})$. Since both the bulk and hydrated water can contribute to intermolecular stretching vibration, the concentration dependence of $\omega_{\mathrm{S}}$ and $\gamma_{\mathrm{S}}$ can be attributed to changes in hydrated relative to bulk water dynamics. A small 3\% increase in the resonant frequency represents that the average $\mathrm{O} \cdots \mathrm{O}$ distances of the bulk and hydrated water are quite similar, and the HB longitudinal modulus (Young's modulus) of the bulk is not drastically different from that of the hydrated water. ${ }^{85}$ This observation is also in agreement with MD simulations, where the $\mathrm{O} \cdots \mathrm{O}$ RDF around the glucose hydroxyl oxygen indicated that the water-water distance is approximately equal to the glucose-water distance. ${ }^{6}$ The larger increase in $\gamma_{\mathrm{S}}$ aligns with the effect of temperature increase, ${ }^{86}$ where the tetrahedral HB coordination is more distorted at higher temperatures. Since the larger damping constant yields a broader absorption band, representing diverse intermolecular quantum states with various $\mathrm{HB}$ distances and $\mathrm{HB}$ angles, ${ }^{83}$ this result is interpreted as the distortion of the HB tetrahedron. ${ }^{41,42}$ This interpretation coincides with Raman scattering ${ }^{8-10}$ and MD simulations. ${ }^{5,6}$ Lee et $a l .{ }^{6}$ used a classical MD simulation to introduce the $q$ factor (tetrahedral order parameter ${ }^{73}$ ) and then found that a low- $q$ (distorted tetrahedral structure) component increases within the first hydration shell, while the $q$ value in the second hydration shell comes very close to that of bulk water. Recent $a b$ initio MD simulations have also shown that the local dipole moments of each of the hydroxyl groups in the glucose molecule vary from site to site, concluding that the local HB coordination in the hydration shell is less tetrahedral. ${ }^{62}$

To summarize this section, we remark the destructuring effect on the water HB network implemented by glucose solutes, and as a consequence, it has been found that the fraction of the non-HB water is larger and the tetrahedral $\mathrm{HB}$ alignment shows an inhomogeneous distribution in $\mathrm{HB}$ distances and angles around a glucose surface. Probable reason for such destructuring state is that the steric constraint imposed by the glucose molecule prevents surrounding water molecules 
to form their native tetrahedral structure. ${ }^{6}$ In particular, water molecules around the hydroxyl groups are forced to align in the same direction to form a collapsed tetrahedral structure but are geometrically hampered by the saccharide molecule. This leads to an increase in the population of non-HB water and distorts the HB network in the vicinity of the saccharide molecules. ${ }^{13}$

\section{SUMMARY}

In this work, we determined the broadband complex dielectric constant of the glucose aqueous solutions at $300 \mathrm{~K}$, reflecting various molecular dynamics from the very slow glucose tumbling to the ultrafast $\mathrm{HB}$ dynamics. The complex dielectric constants from $0.5 \mathrm{GHz}$ to $12 \mathrm{THz}$ were decomposed into their constituents, such as the $\beta$ relaxation (glucose tumbling), $\delta$ relaxation (rotation of the hydrated water), slow relaxation (collective reorientation of the $\mathrm{HB}$ bulk water), fast relaxation (relaxation of the non-HB water), intermolecular stretching vibration (hindered translation of the hydrogenbonded water), and intermolecular libration (hindered rotation of HBs). Isolation procedure into each susceptibility component allowed us to separately investigate the dynamics of glucose, hydrated water and bulk water, and to obtain further insights into the hydration state and the destructuring effect on the water HB network, as summarized below.

For the $\beta$ relaxation, the assumption of Stokes-EinsteinDebye type rotational diffusion of a single solute molecule successfully reproduced the effective dipole moment of glucose. Its relative concentration independence (Figure 4) assures that the glucose-glucose dipolar interactions are negligibly small in our investigated concentration regime.

The hydration numbers, $n_{\text {hyd }}$, were evaluated in two ways: one is the " $\delta$ hydration" where all the hydrated water molecules were assumed to contribute to the $\delta$ relaxation mode with a retardation factor $\xi \approx 2.8$ at maximum, and the other is the "whole hydration" where the hydrated water was defined as all the water molecules that do not behave as the bulk water. Consequently, as shown in Figure 7, the whole hydration number was close to the $\delta$ hydration number, indicating no other relaxation components exist except for the $\delta$ relaxation mode. Thus, we can reasonably consider that the hydrated water is moderately retarded and no immobilized or ice-like water with much longer relaxation time was confirmed, as recently reported. ${ }^{6,19}$ The hydration number at the dilution limit, $n_{\text {hyd }}^{0}$ $\approx 21$, represented not only the one directly forming HBs with the glucose polar groups but also the one indirectly perturbed by polar groups and loosely influenced by the van der Waals forces or Coulombic interactions. ${ }^{20}$ Furthermore, the linear decrease in $n_{\text {hyd }}$ was attributed to the overlaps of the hydration shell.

Finally, the HB destructuring effect of glucose solutes was discussed in terms of the population of the non-HB water isolated from the HB network and the structural coordination of water molecules engaging in the HB network. We found, in the vicinity of the glucose surface, the population of the non-HB water was increased and the dynamical tetrahedral structure of the HB network was more disordered with distributed HB distances and angles. These experimental results were consistent with concept of the HB destructuring effect previously proposed by simulations $s^{6,7}$ and experiments ${ }^{8-10,72,85}$ and produced a more clear image of how solute molecules destructure the water HBs.

We can thus conclude that the broadband complex dielectric constant is an appropriate tool to unveil the ambiguously revealed water-saccharide interactions, which is supposed to be an origin of roles and characteristics of saccharides in biosystems, such as resistance to thermal and dehydration stresses. Therefore, further investigations of systematical comparison among various saccharides or more complex water-saccharide-protein systems will describe a more detailed picture about mechanisms of saccharides in the biological environment.

\section{ACKNOWLEDGMENTS}

We are grateful to Mr. Motoki Imamura and Mr. Akiyoshi Irisawa (ADVANTEST Corporation, Japan) for their technical supports. We also acknowledge Professor Garry John Piller (Graduate School of Agriculture, Kyoto University, Japan) for his help and useful discussions. Financial support was provided by Industry-Academia Collaborative R\&D from Japan Science and Technology Agency and JSPS KAKENHI Grant No. 26295.

${ }^{1}$ T. Arakawa and S. N. Timashef, Biochemistry 21, 6536 (1982).

${ }^{2}$ M. Fernández-Alonso, F. J. Cañada, J. Jiménez-Barbero, and G. J. Cuevas, J. Am. Chem. Soc. 127, 7379 (2005).

${ }^{3}$ A. Magno and P. Gallo, J. Phys. Chem. Lett. 2, 977 (2011).

${ }^{4}$ C. J. Roberts and P. G. Debenedetti, J. Phys. Chem. B 103, 7308 (1999).

${ }^{5}$ P. Bordat, A. Lerbret, J.-P. Demaret, F. Affouard, and M. Descapms, Europhys. Lett. 65, 41 (2004).

${ }^{6}$ S. L. Lee, P. G. Debenedetti, and J. R. Errington, J. Chem. Phys. 122, 204511 (2005).

${ }^{7}$ A. Lerbret, P. Bordat, F. Affouard, M. Descamps, and F. Migliardo, J. Phys. Chem. B 109, 11046 (2005)

${ }^{8}$ C. Branca, S. Magazù, G. Maisano, and P. Migliardo, J. Chem. Phys. 111, 281 (1999).

${ }^{9}$ C. Branca, S. Magazù, G. Maisano, S. M. Bennington, and B. Fåk, J. Phys. Chem. B 107, 1444 (2003).

${ }^{10}$ A. Lerbret, P. Bordat, F. Affouard, Y. Guinet, A. Hédoux, L. Paccou, D. Prevost, and M. Descamps, Carbohydr. Res. 340, 881 (2005).

${ }^{11}$ P. Ball, Chem. Rev. 108, 74 (2008).

${ }^{12}$ B. Bagchi, Chem. Rev. 105, 3197 (2005).

${ }^{13}$ L. Zhao, K. Ma, and Z. Yang, Int. J. Mol. Sci. 16, 8454 (2015).

${ }^{14}$ D. Fioretto, L. Comez, M. E. Gallina, A. Moressi, L. Palamieri, M. Paolantoni, P. Sassi, and F. Scarponi, Chem. Phys. Lett. 441, 232 (2007).

${ }^{15}$ M. Paolantoni, P. Sassi, A. Morresi, and S. Santini, J. Chem. Phys. 127, 024504 (2007)

${ }^{16}$ M. Paolantoni, L. Comez, M. Gallina, P. Sassi, F. Scarponi, D. Fioretto, and A. Morresi, J. Phys. Chem. B 113, 7874 (2009).

${ }^{17}$ L. Lupi, L. Comez, M. Paolantoni, D. Fioretto, and B. M. Ladanyi, J. Phys. Chem. B 116, 7499 (2012).

${ }^{18}$ L. Lupi, L. Comez, M. Paolantoni, S. Perticaroli, P. Sassi, A. Morresi, B. M. Ladanyi, and D. Fioretto, J. Phys. Chem. B 116, 14760 (2012).

${ }^{19}$ L. R. Winther, J. Qvist, and B. Halle, J. Phys. Chem. B 116, 9196 (2012).

${ }^{20}$ P. E. Mason, G. W. Neilson, J. E. Enderby, M.-L. Saboungi, and J. W. Brady, J. Phys. Chem. B 100, 13104 (2005).

${ }^{21}$ C. C. M. Groot and H. J. Bakker, J. Chem. Phys. 140, 234503 (2014).

${ }^{22}$ S. Mashimo, N. Miura, and T. Umehara, J. Chem. Phys. 97, 7659 (1992).

${ }^{23}$ K. Fuchs and U. Kaatze, J. Phys. Chem. B 105, 2036 (2001).

${ }^{24}$ K. Fuchs and U. Kaatze, J. Chem. Phys. 116, 7137 (2002).

${ }^{25}$ G. Otting, Prog. Nucl. Magn. Reson. Spectrosc. 31, 259 (1997).

${ }^{26}$ J. Bergmann, D. Nordlund, Ph. Wernet, M. Odelius, L. G. M. Pettersson, and A. Nilsson, Phys. Rev. E 76, 024202 (2007).

${ }^{27}$ A. K. Soper and C. Benmore, J. Phys. Rev. Lett. 101, 065502 (2008).

${ }^{28}$ I. Ohmine and H. Tanaka, Chem. Rev. 93, 2545 (1993). 
${ }^{29}$ C. P. Lawrence and J. L. Skinner, J. Chem. Phys. 118, 264 (2003).

${ }^{30}$ T. Arikawa, M. Nagai, and K. Tanaka, Chem. Phys. Lett. 457, 12 (2008).

${ }^{31}$ K. Shiraga, T. Suzuki, N. Kondo, J. De Baerdemaeker, and Y. Ogawa, Carbohydr. Res. 406, 46 (2015).

${ }^{32}$ M. Heyden, E. Bründermann, U. Heugen, G. Niehues, D. M. Leitner, and M. Havenith, J. Am. Chem. Soc. 130, 5773 (2008).

${ }^{33}$ U. Heugen, G. Schwaab, E. Bründermann, M. Heyden, X. Yu, D. M. Leinter, and M. Havenith, Proc. Natl. Acad. Sci. U. S. A. 103, 12301 (2006).

${ }^{34}$ H. Yada, M. Nagai, and K. Takana, Chem. Phys. Lett. 464, 166 (2008).

${ }^{35}$ H. Yada, M. Nagai, and K. Takana, Chem. Phys. Lett. 473, 279 (2009).

${ }^{36}$ R. Buchner, J. Barthel, and J. Stauber, Chem. Phys. Lett. 306, 57 (1999).

${ }^{37}$ T. Yasui, E. Saneyoshi, and T. Araki, Appl. Phys. Lett. 87, 061101 (2005).

${ }^{38}$ A. Bartels, R. Cerna, C. Kistner, A. Thoma, F. Hudert, C. Janke, and T. Dekorsy, Rev. Sci. Instrum. 78, 035107 (2007).

${ }^{39}$ M. Nagai, H. Yada, T. Arikawa, and K. Tanaka, Int. J. Infrared Millimeter Waves 27, 505 (2006).

${ }^{40}$ J. E. Bertie and Z. Lan, J. Chem. Phys. 105, 8502 (1996).

${ }^{41}$ K. Shiraga, T. Suzuki, N. Kondo, and Y. Ogawa, J. Chem. Phys. 141, 235103 (2014).

${ }^{42}$ K. Shiraga, H. Naito, T. Suzuki, N. Kondo, and Y. Ogawa, J. Phys. Chem. B (in press).

${ }^{43}$ M. Walther, B. M. Fischer, and P. U. Jepsen, Chem. Phys. 288, 261 (2003).

${ }^{44}$ P. C. Upadhya, Y. C. Shen, A. G. Davies, and E. H. Linfield, J. Biol. Phys. 29, 117 (2003).

${ }^{45}$ P. C. Upadhya, Y. C. Shen, A. G. Davies, and E. H. Linfield, Vib. Spectrosc. 35, 139 (2004).

${ }^{46}$ C. Cametti, S. Marchetti, C. M. C Gambi, and G. Onori, J. Phys. Chem. B 115, 7144 (2012).

${ }^{47}$ G. R. Moran, K. R. Jeffrey, J. M. Thomas, and J. R. Stevens, Carbohydr. Res. 328, 573 (2000).

${ }^{48}$ T. R. Noel, S. G. Ring, and M. A. Whittam, J. Phys. Chem. 96, 5662 (1992).

${ }^{49}$ T. R. Noel, R. Parker, and S. G. Ring, Carbohydr. Res. 282, 193 (1996).

${ }^{50} \mathrm{H}$. Weingärtner, A. Knocks, S. Boresch, P. Höchtl, and O. Steinhauser, J. Chem. Phys. 115, 1463 (2001).

${ }^{51}$ E. Grant, R. Sheppard, and G. South, Dielectric Behavior of Biological Molecules in Solution (Clarendon Press, Oxford, UK, 1978).

${ }^{52}$ I. Ohmine, J. Phys. Chem. 99, 6767 (1995).

${ }^{53}$ D. Kivelson, M. G. Kivelson, and I. Oppenheim, J. Chem. Phys. 52, 1810 (1970).

${ }^{54}$ D. Fioretto, L. Comez, S. Corezzi, M. Paolantoni, P. Sassi, and A. Morresi, Food Biophys. 8, 177 (2013).

${ }^{55}$ G. A. Vidulich, D. F. Evans, and R. L. Kay, J. Chem. Phys. 71, 656 (1971).
${ }^{56}$ P. L. Polavarapu and C. S. Ewig, J. Comput. Chem. 13, 1255 (1992).

${ }^{57}$ F. Franks, D. S. Reid, and A. Suggett, J. Solution Chem. 2, 99 (1973).

${ }^{58}$ R. L. Fulton, J. Chem. Phys. 136, 084502 (2012)

${ }^{59}$ R. Pethig, Annu. Rev. Phys. Chem. 43, 177-205 (1992).

${ }^{60}$ A. Oleinikova, P. Sasisanker, and H. Weingärtner, J. Phys. Chem. B 108, 8467 (2004).

${ }^{61}$ M. J. Tait, A. Suggett, F. Franks, S. Ablett, and P. A. Quickenden, J. Solution Chem. 1, 131 (1972).

${ }^{62}$ T. Suzuki, Phys. Chem. Chem. Phys. 10, 96 (2008).

${ }^{63}$ S. Mashimo, S. Kuwabara, S. Yagihara, and K. Higashi, J. Phys. Chem. 91, 6337 (1987).

${ }^{64}$ S. Perticaroli, M. Nakanishi, E. Pashkovski, and A. P. Sokolov, J. Phys. Chem. B 117, 7729 (2013).

${ }^{65}$ D. R. Martin and D. V. Matyushov, J. Chem. Phys. 141, 22D502 (2014).

${ }^{66}$ H. Shiio, J. Am. Chem. Soc. 80, 70 (1958).

${ }^{67}$ S. A. Galema and H. Hoeiland, J. Phys. Chem. 95, 5321 (1991).

${ }^{68}$ T. Furuki, Carbohydr. Res. 337, 441 (2002).

${ }^{69}$ D. M. Leitner, M. Gruebele, and M. Havenith, HFSP J. 2 , 314 (2008).

${ }^{70}$ C. Molteni and M. Parrinello, J. Am. Chem. Soc. 120, 2168 (1998).

${ }^{71}$ A. Stradner, H. Sedgwick, F. Cardinaux, W. C. K. Poon, S. U. Egelhaaf, and P. Schurtenberger, Nature 432, 492 (2004).

${ }^{72}$ J. D. Batchelor, A. Olteanu, A. Tripathy, and G. J. Pielak, J. Am. Chem. Soc. 126, 1958 (2004).

${ }^{73}$ J. E. Errington, P. G. Debenedetti, and S. Torquato, Phys. Rev. Lett. 89, 215503 (2002).

${ }^{74}$ A. Godec, J. C. Smith, and F. Merzel, Phys. Rev. Lett. 107, 267801 (2011).

${ }^{75}$ S. E. Pagnotta, S. E. McLain, A. K. Soper, F. Bruni, and M. A. Ricci, J. Phys. Chem. B 114, 4904 (2010).

${ }^{76}$ G. E. Walrafen, J. Chem. Phys. 40, 3249 (1964).

${ }^{77}$ M. Sharma, R. Resta, and R. Car, Phys. Rev. Lett. 95, 187401 (2005).

${ }^{78}$ H. Torii, J. Phys. Chem. B 115, 6636 (2011).

${ }^{79}$ H. Torii, J. Chem. Theory Comput. 10, 1219 (2014).

${ }^{80}$ G. E. Walrafen, Y. C. Chu, and G. J. Piermarini, J. Phys. Chem. 100, 10363 (1996).

${ }^{81}$ T. Tassaing, Y. Danten, and M. Besnard, J. Mol. Liq. 101, 149 (2002).

${ }^{82}$ J. Martí, J. A. Padro, and E. Guàrdia, J. Chem. Phys. 105, 639 (1996).

${ }^{83}$ J. Martí, Phys. Rev. E 61, 449 (2000).

${ }^{84}$ H. Ma and J. Ma, J. Chem. Phys. 135, 054504 (2011).

${ }^{85}$ S. Perticaroli, P. Sassi, A. Moressi, and M. Paolantoni, J. Raman Spectrosc. 39, 227 (2008).

${ }^{86}$ H. R. Zelsmann, J. Mol. Struct. 350, 95 (1995). 